\title{
Existence of Bounded Positive Solutions for Partial Difference Equations with Delays
}

\author{
Zeqing Liu, ${ }^{1}$ Zhihua Wu, $^{1}$ Young Chel Kwun, ${ }^{2}$ \\ and Shin Min Kang ${ }^{3}$ \\ ${ }^{1}$ Department of Mathematics, Liaoning Normal University, Dalian, Liaoning 116029, China \\ ${ }^{2}$ Department of Mathematics, Dong-A University, Busan 614-714, Republic of Korea \\ ${ }^{3}$ Department of Mathematics and RINS, Gyeongsang National University, Jinju 660-701, Republic of Korea \\ Correspondence should be addressed to Young Chel Kwun, yckwun@dau.ac.kr
}

Received 3 February 2012; Accepted 14 March 2012

Academic Editor: Agacik Zafer

Copyright (c) 2012 Zeqing Liu et al. This is an open access article distributed under the Creative Commons Attribution License, which permits unrestricted use, distribution, and reproduction in any medium, provided the original work is properly cited.

This paper deals with solvability of the third-order nonlinear partial difference equation with delays $\Delta_{n}\left(a_{m, n} \Delta_{m}^{2}\left(x_{m, n}+b_{m, n} x_{m-\tau_{0}, n-\sigma_{0}}\right)\right)+f\left(m, n, x_{m-\tau_{1, m}, n-\sigma_{1, n}}, \ldots, x_{m-\tau_{k, m}, n-\sigma_{k, n}}\right)=c_{m, n}, m \geq m_{0}, n \geq$ $n_{0}$. With the help of the Banach fixed-point theorem, the existence results of uncountably many bounded positive solutions for the partial difference equation are given; some Mann iterative schemes with errors are suggested, and the error estimates between the iterative schemes and the bounded positive solutions are discussed. Three nontrivial examples illustrating the results presented in this paper are also provided.

\section{Introduction and Preliminaries}

In the past twenty years many authors studied the oscillation, nonoscillation, asymptotic behavior, and solvability for various neutral delay difference and partial difference equations; see, for example, [1-14] and the references cited therein.

By using the Banach fixed-point theorem, Cheng [2] investigated the existence of a nonoscillatory solution for the second-order neutral delay difference equation with positive and negative coefficients

$$
\Delta^{2}\left(x_{n}+p x_{n-m}\right)+p_{n} x_{n-k}-q_{n} x_{n-l}=0, \quad n \geq n_{0}
$$


under the condition $p \in \mathbb{R} \backslash\{-1\}$. Applying a nonlinear alternative of Leray-Schauder type for condensing operators, Agarwal et al. [1] discussed the existence of a bounded nonoscillatory solution for the discrete equation:

$$
\Delta\left(a_{n} \Delta\left(x_{n}+p x_{n-\tau}\right)\right)+F\left(n+1, x_{n+1-\sigma}\right)=0, \quad n \geq 0 .
$$

Liu et al. [6] introduced the second-order nonlinear neutral delay difference equation

$$
\Delta\left(a_{n} \Delta\left(x_{n}+b x_{n-\tau}\right)\right)+f\left(n, x_{n-d_{1 n}}, x_{n-d_{2 n}}, \ldots, x_{n-d_{k n}}\right)=c_{n}, \quad n \geq 0
$$

with respect to all $b \in \mathbb{R}$ and gave the existence of uncountably many bounded nonoscillatory solutions for (1.3) by utilizing the Banach fixed-point theorem. Kong et al. [3] investigated a class of BVPs for the third-order functional difference equation

$$
\Delta^{3} x_{n}+a_{n} f\left(n, x_{w(n)}\right)=0, \quad n \geq 0
$$

and established the existence of positive solutions for (1.4) under certain conditions. Using the Schauder fixed-point theorem, Yan and Liu [12] studied the existence of a bounded nonoscillatory solution for third order nonlinear delay difference equation

$$
\Delta^{3} x_{n}+f\left(n, x_{n}, x_{n-r}\right)=0, \quad n \geq n_{0}
$$

and provided also a necessary and sufficient condition for the existence of a bounded nonoscillatory solution of (1.5).

Karpuz and Öcalan [4] discussed the first-order linear partial difference equation:

$$
x_{m+1, n}+x_{m, n+1}-x_{m, n}+p_{m, n} x_{m-k, n-l}=0, \quad(m, n) \in \mathbb{Z}_{0,0},
$$

where $\left\{p_{m, n}\right\}_{(m, n) \in \mathbb{Z}_{0,0}}$ is a nonnegative sequence and $k, l \in \mathbb{N}_{1}$ and obtained sufficient conditions under which every solution of (1.6) is oscillatory. Yang and Zhang [14] considered oscillations of the partial difference equation with several nonlinear terms of the form

$$
x_{m+1, n}+x_{m, n+1}-x_{m, n}+\sum_{i=1}^{h} p_{i}(m, n)\left|x_{m-k_{i}, n-l_{i}}\right|^{\alpha_{i}} \operatorname{sgn} x_{m-k_{i}, n-l_{i}}=0
$$

and established some new oscillatory criteria by making use of frequency measures. Wong and Agarwal [10] considered the partial difference equations

$$
\begin{gathered}
x_{m+1, n}+\beta_{m, n} x_{m, n+1}-\delta_{m, n} x_{m, n}+p\left(m, n, x_{m-k, n-l}\right)=Q\left(m, n, x_{m-k, n-l}\right), \quad m \geq m_{0}, n \geq n_{0}, \\
x_{m+1, n}+\beta_{m, n} x_{m, n+1}-\delta_{m, n} x_{m, n}+\sum_{i=1}^{\tau} p_{i}\left(m, n, x_{m-k, n-l}\right)=\sum_{i=1}^{\tau} Q\left(m, n, x_{m-k, n-l}\right), \quad m \geq m_{0}, n \geq n_{0}
\end{gathered}
$$


and offered sufficient conditions for the oscillation of all solutions for (1.8) and (1.9), respectively. Wong [9] established the existence of eventually positive and monotone decreasing solutions for the partial difference inequalities

$$
\Delta_{m} \Delta_{n} x_{m, n}+\sum_{i=1}^{r} p_{i}\left(m, n, x_{g_{i}(m), h_{i}(n)}\right) \geq(\leq) \sum_{i=1}^{r} Q_{i}\left(m, n, x_{g_{i}(m), h_{i}(n)}\right), \quad m \geq m_{0}, n \geq n_{0},
$$

where $g_{i}(m)$ and $h_{i}(m)$ are some deviating arguments for $1 \leq i \leq \tau$.

However, to the best of our knowledge, there is no literature referred to the following third order nonlinear partial difference equation with delays:

$$
\begin{aligned}
& \Delta_{n}\left(a_{m, n} \Delta_{m}^{2}\left(x_{m, n}+b_{m, n} x_{m-\tau_{0}, n-\sigma_{0}}\right)\right)+f\left(m, n, x_{m-\tau_{1, m}, n-\sigma_{1, n}}, \ldots, x_{m-\tau_{k, m}, n-\sigma_{k, n}}\right) \\
& \quad=c_{m, n}, \quad m \geq m_{0}, n \geq n_{0},
\end{aligned}
$$

where $m_{0}, n_{0} \in \mathbb{N}_{0}, k, \tau_{0}, \sigma_{0} \in \mathbb{N},\left\{a_{m, n}\right\}_{(m, n) \in \mathbb{N}_{m_{0}, n_{0}}},\left\{b_{m, n}\right\}_{(m, n) \in \mathbb{N}_{m_{0}, n_{0}}}\left\{c_{m, n}\right\}_{(m, n) \in \mathbb{N}_{m_{0}, n_{0}}}$ are real sequences with $a_{m, n} \neq 0, b_{m, n} \neq \pm 1$ for $(m, n) \in \mathbb{N}_{m_{0}, n_{0}}, f: \mathbb{N}_{m_{0}, n_{0}} \times \mathbb{R}^{k} \rightarrow \mathbb{R}$ and $\left\{\tau_{l, m}, \sigma_{l, n}\right.$ : $\left.(m, n) \in \mathbb{N}_{m_{0}, n_{0}}, l \in\{1,2, \ldots, k\}\right\} \subseteq \mathbb{Z}$ with

$$
\lim _{m \rightarrow \infty}\left(m-\tau_{l, m}\right)=\lim _{n \rightarrow \infty}\left(n-\sigma_{l, n}\right)=+\infty, \quad l \in\{1,2, \ldots, k\} .
$$

The aim of this paper is to establish three sufficient conditions of the existence of uncountably many bounded positive solutions for (1.11) by using the Banach fixed-point theorem, to suggest some Mann iterative methods with errors for these bounded positive solutions and to compute the error estimates between the bounded positive solutions and the sequences generated by the Mann iterative methods with errors. In order to explain the results presented in this paper, three nontrivial examples are constructed.

Throughout this paper, the forward partial difference operators $\Delta_{m}$ and $\Delta_{n}$ are defined by $\Delta_{m} x_{m, n}=x_{m+1, n}-x_{m, n}$ and $\Delta_{n} x_{m, n}=x_{m, n+1}-x_{m, n}$, respectively the second and thirdorder partial difference operators are defined by $\Delta_{m}^{2} x_{m, n}=\Delta_{m}\left(\Delta_{m} x_{m, n}\right)$ and $\Delta_{n} \Delta_{m}^{2} x_{m, n}=$ $\Delta_{n}\left(\Delta_{m}^{2} x_{m, n}\right)$, respectively. Let $\mathbb{R}=(-\infty,+\infty), \mathbb{N}$ and $\mathbb{Z}$ denote the sets of all positive integers and integers, respectively,

$$
\begin{gathered}
\mathbb{N}_{0}=\{0\} \cup \mathbb{N}, \mathbb{N}_{s}=\left\{n: n \in \mathbb{N}_{0} \text { with } n \geq s\right\}, \quad s \in \mathbb{N}_{0}, \\
\mathbb{N}_{s, t}=\left\{(m, n): m, n \in \mathbb{N}_{0} \text { with } m \geq s, n \geq t\right\}, \quad s, t \in \mathbb{N}_{0}, \\
\mathbb{Z}_{s, t}=\{(m, n): m, n \in \mathbb{Z} \text { with } m \geq s, n \geq t\}, \quad s, t \in \mathbb{Z}, \\
\alpha=\min \left\{m-\tau_{0}, m-\tau_{l, m}: 1 \leq l \leq k, m \in \mathbb{N}_{m_{0}}\right\}, \\
\beta=\min \left\{n-\sigma_{0}, n-\sigma_{l, n}: 1 \leq l \leq k, n \in \mathbb{N}_{n_{0}}\right\} .
\end{gathered}
$$


$l_{\alpha, \beta}^{\infty}$ represents the Banach space of all bounded sequences on $\mathbb{Z}_{\alpha, \beta}$ with the norm

$$
\begin{gathered}
\|x\|=\sup _{m, n \in \mathbb{Z}_{\alpha, \beta}}\left|x_{m, n}\right| \text { for } x=\left\{x_{m, n}\right\}_{(m, n) \in \mathbb{Z}_{\alpha, \beta}} \in l_{\alpha, \beta^{\prime}}^{\infty} \\
A(N, M)=\left\{x=\left\{x_{m, n}\right\}_{(m, n) \in \mathbb{Z}_{\alpha, \beta}} \in l_{\alpha, \beta}^{\infty}: N \leq x_{m, n} \leq M,(m, n) \in \mathbb{Z}_{\alpha, \beta}\right\} \quad \text { for } M>N>0 .
\end{gathered}
$$

It is not difficult to see that $A(N, M)$ is a bounded closed and convex subset of the Banach space $l_{\alpha, \beta}^{\infty}$. By a solution of (1.11), we mean a sequence $\left\{x_{m, n}\right\}_{(m, n) \in \mathbb{Z}_{\alpha, \beta}}$ with positive integers $m_{1} \geq m_{0}+\tau_{0}+|\alpha|$ and $n_{1} \geq n_{0}+\sigma_{0}+|\beta|$ such that (1.11) is satisfied for all $m \geq m_{1}$ and $n \geq n_{1}$.

Lemma 1.1 (see [15]). Let $\{\alpha(n)\}_{n \in \mathbb{N}_{0}},\{\beta(n)\}_{n \in \mathbb{N}_{0}},\{\gamma(n)\}_{n \in \mathbb{N}_{0}}$, and $\{t(n)\}_{n \in \mathbb{N}_{0}}$ be nonnegative sequences satisfying the inequality

$$
\alpha(n+1) \leq(1-t(n)) \alpha(n)+t(n) \beta(n)+\gamma(n), \quad n \in \mathbb{N}_{0},
$$

where $\{t(n)\}_{n \in \mathbb{N}_{0}} \subset[0,1]$ with $\sum_{n=0}^{\infty} t(n)=+\infty, \lim _{n \rightarrow \infty} \beta(n)=0$ and $\sum_{n=0}^{\infty} \gamma(n)<+\infty$. Then $\lim _{n \rightarrow \infty} \alpha(n)=0$.

\section{Existence of Uncountably Many Bounded Positive Solutions and Mann Iterative Schemes with Errors}

Utilizing the Banach fixed-point theorem, we now investigate the existence of uncountably many bounded positive solutions for (1.11), suggest the Mann type iterative schemes with errors and discuss the error estimates between the bounded positive solutions and the sequences generated by the Mann iterative schemes.

Theorem 2.1. Assume that there exists positive constants $M$ and $N$, nonnegative constants $b_{1}$ and $b_{2}$, and nonnegative sequences $\left\{P_{m, n}\right\}_{(m, n) \in \mathbb{N}_{m_{0}, n_{0}}}$ and $\left\{Q_{m, n}\right\}_{(m, n) \in \mathbb{N}_{m_{0}, n_{0}}}$ satisfying

$$
\begin{gathered}
b_{1}+b_{2}<1, \quad N<\left[1-\left(b_{1}+b_{2}\right)\right] M, \\
-b_{2} \leq b_{m, n} \leq b_{1}, \quad \text { eventually, } \\
\left|f\left(m, n, u_{1}, u_{2}, \ldots, u_{k}\right)-f\left(m, n, \bar{u}_{1}, \bar{u}_{2}, \ldots, \bar{u}_{k}\right)\right| \leq P_{m, n} \max \left\{\left|u_{l}-\bar{u}_{l}\right|: 1 \leq l \leq k\right\}, \\
\left(m, n, u_{l}, \bar{u}_{l}\right) \in \mathbb{N}_{m_{0}, n_{0}} \times[N, M]^{2}, \quad 1 \leq l \leq k, \\
\left|f\left(m, n, u_{1}, u_{2}, \ldots, u_{k}\right)\right| \leq Q_{m, n}, \quad\left(m, n, u_{l}\right) \in \mathbb{N}_{m_{0}, n_{0}} \times[N, M], 1 \leq l \leq k ; \\
\sum_{j=m_{0}}^{\infty} \sum_{i=j}^{\infty} \sup _{n \in \mathbb{N}_{n_{0}}}\left\{\frac{1}{\left|a_{i, n}\right|} \sum_{t=n}^{\infty} \max \left\{P_{i, t}, Q_{i, t},\left|c_{i, t}\right|\right\}\right\}<+\infty .
\end{gathered}
$$


Then

(a) for any $L \in\left(N+b_{1} M,\left(1-b_{2}\right) M\right)$, there exist $\theta \in(0,1), m_{1} \geq m_{0}+\tau_{0}+|\alpha|$ and $n_{1} \geq$ $n_{0}+\sigma_{0}+|\beta|$ such that for any $x(0)=\left\{x_{m, n}(0)\right\}_{(m, n) \in \mathbb{Z}_{\alpha, \beta}} \in A(N, M)$, the Mann iterative sequence with errors $\{x(s)\}_{s \in \mathbb{N}_{0}}=\left\{x_{m, n}(s)\right\}_{(m, n, s) \in \mathbb{Z}_{\alpha, \beta} \times \mathbb{N}_{0}}$ generated by the scheme:

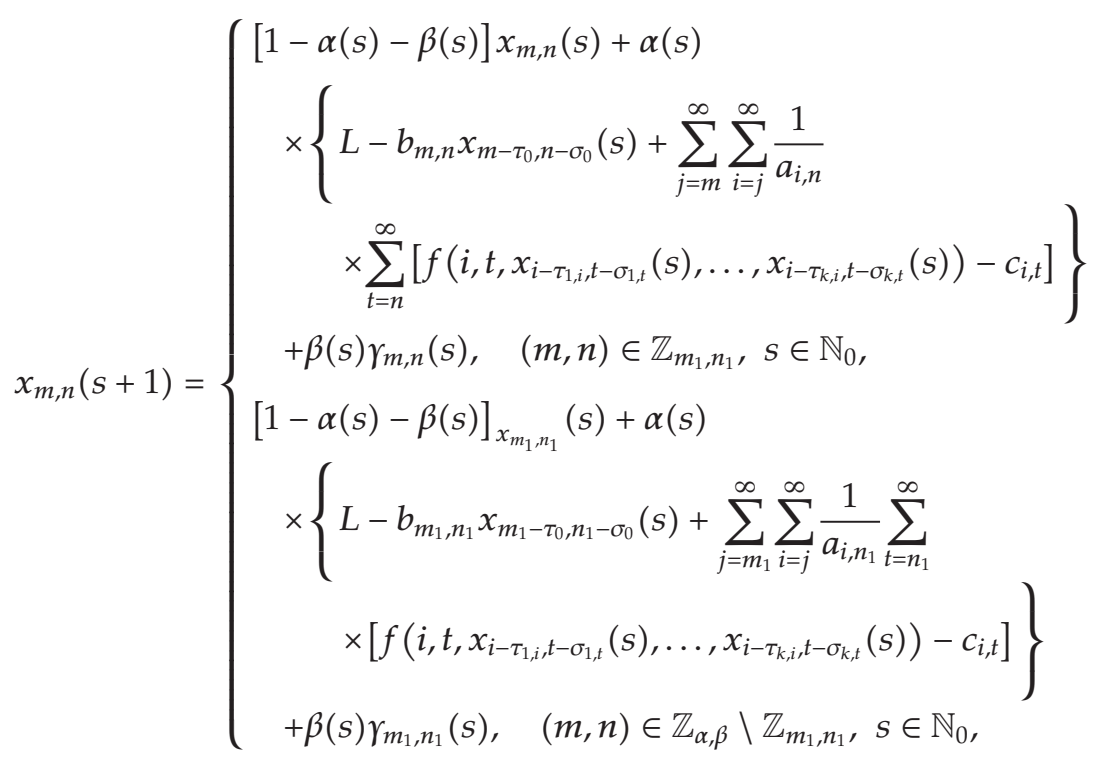

converges to a bounded positive solution $x \in A(M, N)$ of (1.11) and has the following error estimate:

$$
\|x(s+1)-x\| \leq[1-(1-\theta) \alpha(s)]\|x(s)-x\|+2 M \beta(s), \quad s \in \mathbb{N}_{0},
$$

where $\{\gamma(s)\}_{s \in \mathbb{N}_{0}}$ is an arbitrary sequence in $A(M, N),\{\alpha(s)\}_{s \in \mathbb{N}_{0}}$ and $\{\beta(s)\}_{s \in \mathbb{N}_{0}}$ are any sequences in $[0,1]$ such that

$$
\sum_{s=0}^{\infty} \alpha(s)=+\infty
$$

$\sum_{s=0}^{\infty} \beta(s)<+\infty$ or there exists a sequence $\{\xi(s)\}_{s \in \mathbb{N}_{0}} \subseteq[0,+\infty)$ satisfying

$$
\beta(s)=\xi(s) \alpha(s), \quad s \in \mathbb{N}_{0}, \quad \lim _{s \rightarrow \infty} \xi(s)=0
$$

(b) (1.11) possesses uncountably many bounded positive solutions in $A(M, N)$. 
Proof. First of all we show that (a) holds. Set $L \in\left(N+b_{1} M,\left(1-b_{2}\right) M\right)$. It follows from (2.1), (2.2), and (2.5) that there exist $\theta \in(0,1), m_{1} \geq m_{0}+\tau_{0}+|\alpha|$ and $n_{1} \geq n_{0}+\sigma_{0}+|\beta|$ such that

$$
\begin{gathered}
\theta=b_{1}+b_{2}+\sum_{j=m_{1}}^{\infty} \sum_{i=j}^{\infty} \sup _{n \in \mathbb{N}_{n_{1}}}\left\{\frac{1}{\left|a_{i, n}\right|} \sum_{t=n}^{\infty} P_{i, t}\right\}, \\
-b_{2} \leq b_{m, n} \leq b_{1}, \quad(m, n) \in \mathbb{N}_{m_{1}, n_{1},} \\
\sum_{j=m_{1}}^{\infty} \sum_{i=j}^{\infty} \sup _{n \in \mathbb{N}_{n_{1}}}\left\{\frac{1}{\left|a_{i, n}\right|} \sum_{t=n}^{\infty}\left(Q_{i, t}+\left|c_{i, t}\right|\right)\right\} \leq \min \left\{\left(1-b_{2}\right) M-L, L-b_{1} M-N\right\} .
\end{gathered}
$$

Define a mapping $T_{L}: A(N, M) \rightarrow l_{\alpha, \beta}^{\infty}$ by

$$
T_{L} x_{m, n}=\left\{\begin{array}{l}
L-b_{m, n} x_{m-\tau_{0}, n-\sigma_{0}}+\sum_{j=m}^{\infty} \sum_{i=j}^{\infty} \frac{1}{a_{i, n}} \\
\quad \times \sum_{t=n}^{\infty}\left[f\left(i, t, x_{i-\tau_{1, i}, t-\sigma_{1, t}}, \ldots, x_{i-\tau_{k, i}, t-\sigma_{k, t}}\right)-c_{i, t}\right], \quad(m, n) \in \mathbb{Z}_{m_{1}, n_{1}}, \\
T_{L} x_{m_{1}, n_{1}}, \quad(m, n) \in \mathbb{Z}_{\alpha, \beta} \backslash \mathbb{Z}_{m_{1}, n_{1}}
\end{array}\right.
$$

for each $x=\left\{x_{m, n}\right\}_{(m, n) \in \mathbb{Z}_{\alpha, \beta}} \in A(N, M)$. By employing (2.1)-(2.4) and (2.10)-(2.13), we infer that for $x=\left\{x_{m, n}\right\}_{(m, n) \in \mathbb{Z}_{\alpha, \beta}} y=\left\{y_{m, n}\right\}_{(m, n) \in \mathbb{Z}_{\alpha, \beta}} \in A(N, M)$ and $(m, n) \in \mathbb{Z}_{m_{1}, n_{1}}$

$$
\begin{aligned}
& \left|T_{L} x_{m, n}-T_{L} y_{m, n}\right|=\mid b_{m, n}\left(x_{m-\tau_{0}, n-\sigma_{0}}-y_{m-\tau_{0}, n-\sigma_{0}}\right) \\
& -\sum_{j=m}^{\infty} \sum_{i=j}^{\infty} \frac{1}{a_{i, n}} \sum_{t=n}^{\infty}\left[f\left(i, t, x_{i-\tau_{1, i}, t-\sigma_{1, t}}, \ldots, x_{i-\tau_{k, i}, t-\sigma_{k, t}}\right)\right. \\
& \left.-f\left(i, t, y_{i-\tau_{1, i}, t-\sigma_{1, t}}, \ldots, y_{i-\tau_{k, i}, t-\sigma_{k, t}}\right)\right] \mid \\
& \leq\left|b_{m, n}\right|\left|x_{m-\tau_{0}, n-\sigma_{0}}-y_{m-\tau_{0}, n-\sigma_{0}}\right| \\
& +\sum_{j=m}^{\infty} \sum_{i=j}^{\infty} \frac{1}{\left|a_{i, n}\right|} \sum_{t=n}^{\infty} \mid f\left(i, t, x_{i-\tau_{1, i}, t-\sigma_{1, t}}, \ldots, x_{i-\tau_{k, i}, t-\sigma_{k, t}}\right) \\
& -f\left(i, t, y_{i-\tau_{1, i}, t-\sigma_{1, t}}, \ldots, y_{i-\tau_{k, i}, t-\sigma_{k, t}}\right) \mid \\
& \leq\left(b_{1}+b_{2}\right)\|x-y\|+\sum_{j=m}^{\infty} \sum_{i=j}^{\infty} \frac{1}{\left|a_{i, n}\right|} \\
& \times \sum_{t=n}^{\infty} P_{i, t} \max \left\{\left|x_{i-\tau_{l, i}, t-\sigma_{l, t}}-y_{i-\tau_{l, i}, t-\sigma_{l, t}}\right|: 1 \leq l \leq k\right\}
\end{aligned}
$$


Abstract and Applied Analysis

$$
\begin{aligned}
& \leq\left(b_{1}+b_{2}+\sum_{j=m_{1}}^{\infty} \sum_{i=j}^{\infty} \sup _{n \in \mathbb{N}_{n_{1}}}\left\{\frac{1}{\left|a_{i, n}\right|} \sum_{t=n}^{\infty} P_{i, t}\right\}\right)\|x-y\|=\theta\|x-y\|, \\
T_{L} x_{m, n} & =L-b_{m, n} x_{m-\tau_{0}, n-\sigma_{0}}+\sum_{j=m}^{\infty} \sum_{i=j}^{\infty} \frac{1}{a_{i, n}} \sum_{t=n}^{\infty}\left[f\left(i, t, x_{i-\tau_{1, i}, t-\sigma_{1, t}} \ldots, x_{i-\tau_{k, i}, t-\sigma_{k, t}}\right)-c_{i, t}\right] \\
& \leq L+b_{2} M+\sum_{j=m}^{\infty} \sum_{i=j}^{\infty} \frac{1}{\left|a_{i, n}\right|} \sum_{t=n}^{\infty}\left[\left|f\left(i, t, x_{i-\tau_{1, i}, t-\sigma_{1, t}}, \ldots, x_{i-\tau_{k, i}, t-\sigma_{k, t}}\right)\right|+\left|c_{i, t}\right|\right] \\
& \leq L+b_{2} M+\sum_{j=m_{1}}^{\infty} \sum_{i=j}^{\infty} \sup _{n \in \mathbb{N}_{n_{1}}}\left\{\frac{1}{\left|a_{i, n}\right|} \sum_{t=n}^{\infty}\left(Q_{i, t}+\left|c_{i, t}\right|\right)\right\} \\
& \leq L+b_{2} M+\min \left\{\left(1-b_{2}\right) M-L, L-b_{1} M-N\right\} \leq M, \\
T_{L} x_{m, n} & =L-b_{m, n} x_{m-\tau_{0}, n-\sigma_{0}}+\sum_{j=m}^{\infty} \sum_{i=j}^{\infty} \frac{1}{a_{i, n}} \sum_{t=n}^{\infty}\left[f\left(i, t, x_{i-\tau_{1, i}, t-\sigma_{1, t}}, \ldots, x_{i-\tau_{k, i}, t-\sigma_{k, t}}\right)-c_{i, t}\right] \\
\geq & L-b_{1} M-\sum_{j=m}^{\infty} \sum_{i=j}^{\infty} \frac{1}{\left|a_{i, n}\right|} \sum_{t=n}^{\infty}\left[\left|f\left(i, t, x_{i-\tau_{1, i}, t-\sigma_{1, t}} \ldots, x_{i-\tau_{k, i}, t-\sigma_{k, t}}\right)\right|+\left|c_{i, t}\right|\right] \\
\geq & L-b_{1} M-\sum_{j=m_{1}}^{\infty} \sum_{i=j}^{\infty} \sup _{n \in \mathbb{N}_{n_{1}}}\left\{\frac{1}{\left|a_{i, n}\right|} \sum_{t=n}^{\infty}\left(Q_{i, t}+\left|c_{i, t}\right|\right)\right\} \\
\geq & L-b_{1} M-\min \left\{\left(1-b_{2}\right) M-L, L-b_{1} M-N\right\} \geq N,
\end{aligned}
$$

which lead to

$$
T_{L}(A(N, M)) \subseteq A(N, M), \quad\left\|T_{L} x-T_{L} y\right\| \leq \theta\|x-y\|, \quad x, y \in A(N, M) .
$$

Consequently, (2.15) means that $T_{L}$ is a contraction mapping in $A(N, M)$ and it has a unique fixed-point $x=\left\{x_{m, n}\right\}_{(m, n) \in \mathbb{Z}_{\alpha, \beta}} \in A(N, M)$, which together with (2.13) gives that for $(m, n) \in$ $\mathbb{Z}_{m_{1}, n_{1}}$

$$
x_{m, n}=L-b_{m, n} x_{m-\tau_{0}, n-\sigma_{0}}+\sum_{j=m}^{\infty} \sum_{i=j}^{\infty} \frac{1}{a_{i, n}} \sum_{t=n}^{\infty}\left[f\left(i, t, x_{i-\tau_{1, i}, t-\sigma_{1, t}} \ldots, x_{i-\tau_{k, i}, t-\sigma_{k, t}}\right)-c_{i, t}\right],
$$

which yields that for $(m, n) \in \mathbb{Z}_{m_{1}, n_{1}}$

$$
\begin{gathered}
\Delta_{m}\left(x_{m, n}+b_{m, n} x_{m-\tau_{0}, n-\sigma_{0}}\right)=-\sum_{i=m}^{\infty} \frac{1}{a_{i, n}} \sum_{t=n}^{\infty}\left[f\left(i, t, x_{i-\tau_{1, i}, t-\sigma_{1, t}}, \ldots, x_{i-\tau_{k, i}, t-\sigma_{k, t}}\right)-c_{i, t}\right], \\
\Delta_{m}^{2}\left(x_{m, n}+b_{m, n} x_{m-\tau_{0}, n-\sigma_{0}}\right)=\frac{1}{a_{m, n}} \sum_{t=n}^{\infty}\left[f\left(m, t, x_{m-\tau_{1, m}, t-\sigma_{1, t}} \ldots, x_{m-\tau_{k, m}, t-\sigma_{k, t}}\right)-c_{m, t}\right], \\
\Delta_{n}\left(a_{m, n} \Delta_{m}^{2}\left(x_{m, n}+b_{m, n} x_{m-\tau_{0}, n-\sigma_{0}}\right)\right)=-f\left(m, n, x_{m-\tau_{1, m}, n-\sigma_{1, t}} \ldots, x_{m-\tau_{k, m}, t-\sigma_{k, t}}\right)+c_{m, n},
\end{gathered}
$$

that is, $x=\left\{x_{m, n}\right\}_{(m, n) \in \mathbb{Z}_{\alpha, \beta}}$ is a bounded positive solution of (1.11) in $A(N, M)$. 
Using (2.6), (2.13), and (2.15), we infer that for any $s \in \mathbb{N}_{0}$ and $(m, n) \in \mathbb{Z}_{m_{1}, n_{1}}$

$$
\begin{aligned}
& \left|x_{m, n}(s+1)-x_{m, n}\right|=\mid[1-\alpha(s)-\beta(s)] x_{m, n}(s)+\alpha(s) \\
& \times\left\{L-b_{m, n} x_{m-\tau_{0}, n-\sigma_{0}}(s)\right. \\
& \left.+\sum_{j=m}^{\infty} \sum_{i=j}^{\infty} \frac{1}{a_{i, n}} \sum_{t=n}^{\infty}\left[f\left(i, t, x_{i-\tau_{1, i}, t-\sigma_{1, t}}(s), \ldots, x_{i-\tau_{k, i}, t-\sigma_{k, t}}(s)\right)-c_{i, t}\right]\right\} \\
& +\beta(s) \gamma_{m, n}(s)-x_{m, n} \\
& \leq[1-\alpha(s)-\beta(s)]\left|x_{m, n}(s)-x_{m, n}\right|+\alpha(s)\left|T_{L} x_{m, n}(s)-T_{L} x_{m, n}\right| \\
& +\beta(s)\left|\gamma_{m, n}(s)-x_{m, n}\right| \\
& \leq[1-\alpha(s)-\beta(s)]\|x(s)-x\|+\alpha(s) \theta\|x(s)-x\|+2 M \beta(s) \\
& \leq[1-(1-\theta) \alpha(s)]\|x(s)-x\|+2 M \beta(s),
\end{aligned}
$$

which yields that

$$
\|x(s+1)-x\| \leq[1-(1-\theta) \alpha(s)]\|x(s)-x\|+2 M \beta(s), \quad s \in \mathbb{N}_{0} .
$$

That is, (2.7) holds. Consequently, Lemma 1.1 and (2.7)-(2.9) imply that $\lim _{s \rightarrow \infty} x(s)=x$.

Next we show that (b) holds. Let $L_{1}, L_{2} \in\left(N+b_{1} M,\left(1-b_{2}\right) M\right)$ and let $L_{1} \neq L_{2}$. As in the proof of (a), we infer that for each $i \in\{1,2\}$, there exist $\theta_{i}, m_{i+1}, n_{i+1}$ and $T_{L_{i}}$ satisfying (2.10)(2.13), where $\theta, m_{1}, n_{1}, L$ and $T_{L}$ are replaced by $\theta_{i}, m_{i+1}, n_{i+1}, L_{i}$, and $T_{L_{i}}$, respectively, and the mapping $T_{L_{i}}$ has a fixed-point $x^{i}=\left\{x_{m, n}^{i}\right\}_{(m, n) \in \mathbb{Z}_{\alpha, \beta}} \in A(N, M)$, which is a bounded positive solution of (1.11), that is,

$$
\begin{aligned}
x_{m, n}^{1}= & L_{1}-b_{m, n} x_{m-\tau_{0}, n-\sigma_{0}}^{1} \\
& +\sum_{j=m}^{\infty} \sum_{i=j}^{\infty} \frac{1}{a_{i, n}} \sum_{t=n}^{\infty}\left[f\left(i, t, x_{i-\tau_{1, i}, t-\sigma_{1, t}}^{1} \ldots, x_{i-\tau_{k, i}, t-\sigma_{k, t}}^{1}\right)-c_{i, t}\right], \quad(m, n) \in \mathbb{Z}_{m_{2}, n_{2},} \\
x_{m, n}^{2}= & L_{2}-b_{m, n} x_{m-\tau_{0}, n-\sigma_{0}}^{2} \\
& +\sum_{j=m}^{\infty} \sum_{i=j}^{\infty} \frac{1}{a_{i, n}} \sum_{t=n}^{\infty}\left[f\left(i, t, x_{i-\tau_{1, i}, t-\sigma_{1, t}}^{2} \ldots, x_{i-\tau_{k, i}, t-\sigma_{k, t}}^{2}\right)-c_{i, t}\right], \quad(m, n) \in \mathbb{Z}_{m_{3}, n_{3}} .
\end{aligned}
$$


In order to show that the set of bounded positive solutions of (1.11) is uncountable, it is sufficient to prove that $x^{1} \neq x^{2}$. It follows from (2.3), (2.10), (2.11), (2.20) that for $(m, n) \in$ $\mathbb{Z}_{\max \left\{m_{2}, m_{3}\right\}, \max \left\{n_{2}, n_{3}\right\}}$

$$
\begin{aligned}
& \left|x_{m, n}^{1}-x_{m, n}^{2}\right|=\mid L_{1}-L_{2}-b_{m, n}\left(x_{m-\tau_{0}, n-\sigma_{0}}^{1}-x_{m-\tau_{0}, n-\sigma_{0}}^{2}\right) \\
& +\sum_{j=m}^{\infty} \sum_{i=j}^{\infty} \frac{1}{a_{i, n}} \sum_{t=n}^{\infty}\left[f\left(i, t, x_{i-\tau_{1, i}, t-\sigma_{1, t}}^{1} \ldots, x_{i-\tau_{k, i}, t-\sigma_{k, t}}^{1}\right)\right. \\
& \left.-f\left(i, t, x_{i-\tau_{1, i}, t-\sigma_{1, t}}^{2}, \ldots, x_{i-\tau_{k, i}, t-\sigma_{k, t}}^{2}\right)\right] \\
& \geq\left|L_{1}-L_{2}\right|-\left|b_{m, n}\right|\left|x_{m-\tau_{0}, n-\sigma_{0}}^{1}-x_{m-\tau_{0}, n-\sigma_{0}}^{2}\right| \\
& -\sum_{j=m}^{\infty} \sum_{i=j}^{\infty} \frac{1}{\left|a_{i, n}\right|} \sum_{t=n}^{\infty}\left[\mid f\left(i, t, x_{i-\tau_{1, i}, t-\sigma_{1, t}}^{1}, \ldots, x_{i-\tau_{k, i}, t-\sigma_{k, t}}^{1}\right)\right. \\
& \left.-f\left(i, t, x_{i-\tau_{1, i}, t-\sigma_{1, t}}^{2}, \ldots, x_{i-\tau_{k, i}, t-\sigma_{k, t}}^{2}\right) \mid\right] \\
& \geq\left|L_{1}-L_{2}\right|-\left(b_{1}+b_{2}\right)\left\|x^{1}-x^{2}\right\| \\
& -\sum_{j=m}^{\infty} \sum_{i=j}^{\infty} \frac{1}{\left|a_{i, n}\right|} \sum_{t=n}^{\infty} P_{i, t} \max \left\{\left|x_{i-\tau_{l, i}, t-\sigma_{l, t}}^{1}-x_{i-\tau_{l, i}, t-\sigma_{l, t}}^{2}\right|: 1 \leq l \leq k\right\} \\
& \geq\left|L_{1}-L_{2}\right|-\left(b_{1}+b_{2}+\sum_{j=m}^{\infty} \sum_{i=j}^{\infty} \frac{1}{\left|a_{i, n}\right|} \sum_{t=n}^{\infty} P_{i, t}\right)\left\|x^{1}-x^{2}\right\| \\
& \geq\left|L_{1}-L_{2}\right|-\left(b_{1}+b_{2}+\sum_{j=\max \left\{m_{2}, m_{3}\right\}}^{\infty} \sum_{i=j}^{\infty} \sup _{n \in \mathbb{N}_{\max \left\{n_{2}, n_{3}\right\}}}\left\{\frac{1}{\left|a_{i, n}\right|} \sum_{t=n}^{\infty} P_{i, t}\right\}\right)\left\|x^{1}-x^{2}\right\| \\
& \geq\left|L_{1}-L_{2}\right|-\max \left\{\theta_{1}, \theta_{2}\right\}\left\|x^{1}-x^{2}\right\|,
\end{aligned}
$$

which implies that

$$
\left\|x^{1}-x^{2}\right\| \geq \frac{\left|L_{1}-L_{2}\right|}{1+\max \left\{\theta_{1}, \theta_{2}\right\}}>0,
$$

that is, $x^{1} \neq x^{2}$. This completes the proof.

Theorem 2.2. Assume that there exist positive constants $M$ and $N$, negative constants $b_{1}$ and $b_{2}$ and nonnegative sequences $\left\{P_{m, n}\right\}_{(m, n) \in \mathbb{N}_{m_{0}, n_{0}}}$ and $\left\{Q_{m, n}\right\}_{(m, n) \in \mathbb{N}_{m_{0}, n_{0}}}$ satisfying (2.3)-(2.5) and

$$
\begin{gathered}
b_{1}<-1, \quad N\left(1+b_{2}\right)>M\left(1+b_{1}\right) ; \\
b_{2} \leq b_{m, n} \leq b_{1}, \quad \text { eventually. }
\end{gathered}
$$


Then

(a) for any $L \in\left(M\left(1+b_{1}\right), N\left(1+b_{2}\right)\right)$, there exist $\theta \in(0,1), m_{1} \geq m_{0}+\tau_{0}+|\alpha|$ and $n_{1} \geq n_{0}+\sigma_{0}+|\beta|$ such that for each $x(0)=\left\{x_{m, n}(0)\right\}_{(m, n) \in \mathbb{Z}_{\alpha, \beta}} \in A(N, M)$, the Mann iterative sequence with errors $\{x(s)\}_{s \in \mathbb{N}_{0}}=\left\{x_{m, n}(s)\right\}_{(m, n, s) \in \mathbb{Z}_{\alpha, \beta} \times \mathbb{N}_{0}}$ generated by the scheme:

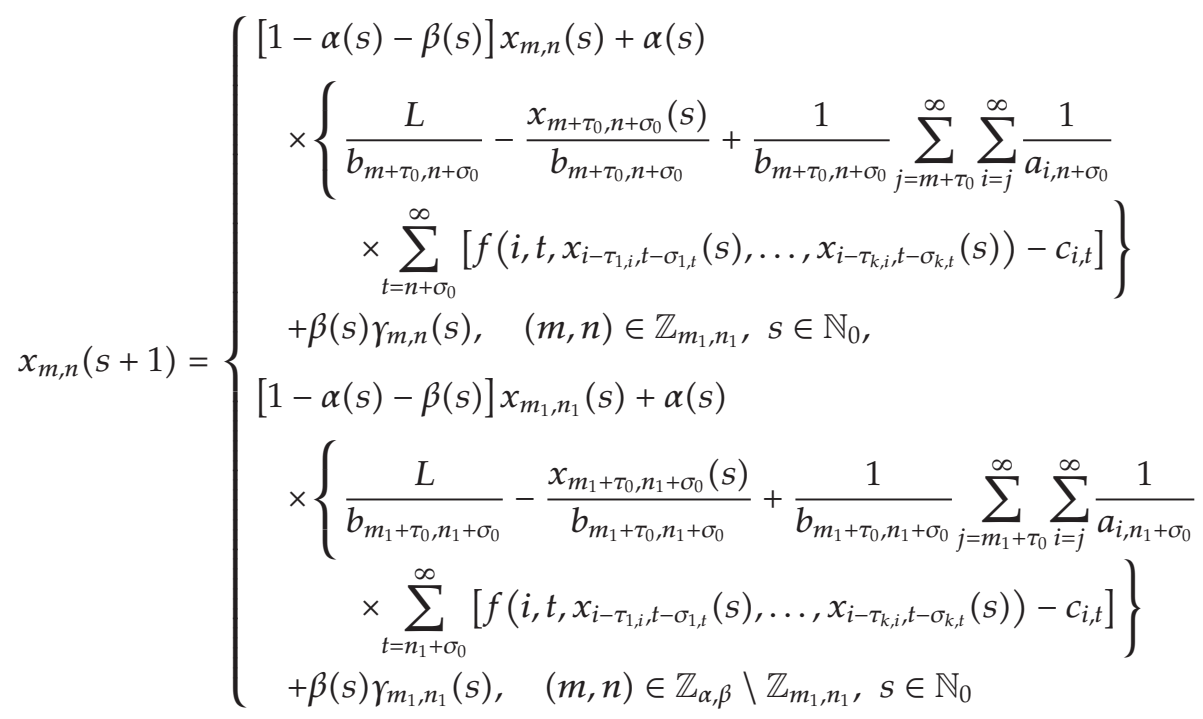

converges to a bounded positive solution $x \in A(N, M)$ of (1.11) and has the error estimate (2.7), where $\{\gamma(s)\}_{s \in \mathbb{N}_{0}}$ is an arbitrary sequence in $A(N, M),\{\alpha(s)\}_{s \in \mathbb{N}_{0}}$ and $\{\beta(s)\}_{s \in \mathbb{N}_{0}}$ are any sequences in $[0,1]$ satisfying (2.8) and (2.9);

(b) (1.11) possesses uncountably many bounded positive solutions in $A(M, N)$.

Proof. First of all we show (a). Taking $L \in\left(M\left(1+b_{1}\right), N\left(1+b_{2}\right)\right)$, from (2.5), (2.23), and (2.24) we infer that there exist $\theta \in(0,1), m_{1} \geq m_{0}+\tau_{0}+|\alpha|$ and $n_{1} \geq n_{0}+\sigma_{0}+|\beta|$ such that

$$
\begin{gathered}
\theta=-\frac{1}{b_{1}}\left(1+\sum_{j=m_{1}}^{\infty} \sum_{i=j}^{\infty} \sup _{n \in \mathbb{N}_{n_{1}}}\left\{\frac{1}{\left|a_{i, n}\right|} \sum_{t=n}^{\infty} P_{i, t}\right\}\right), \\
b_{2} \leq b_{m, n} \leq b_{1}, \quad(m, n) \in \mathbb{N}_{m_{1}, n_{1},} \\
\sum_{j=m_{1}}^{\infty} \sum_{i=j}^{\infty} \sup _{n \in \mathbb{N}_{n_{1}}}\left\{\frac{1}{\left|a_{i, n}\right|} \sum_{t=n}^{\infty}\left(Q_{i, t}+\left|c_{i, t}\right|\right)\right\} \leq \min \left\{L-M\left(1+b_{1}\right), b_{1} N\left(1+\frac{1}{b_{2}}\right)-\frac{b_{1} L}{b_{2}}\right\} .
\end{gathered}
$$

Define a mapping $T_{L}: A(N, M) \rightarrow l_{\alpha, \beta}^{\infty}$ by

$$
T_{L} x_{m, n}=\left\{\begin{array}{l}
\frac{L}{b_{m+\tau_{0}, n+\sigma_{0}}}-\frac{x_{m+\tau_{0}, n+\sigma_{0}}}{b_{m+\tau_{0}, n+\sigma_{0}}}+\frac{1}{b_{m+\tau_{0}, n+\sigma_{0}}} \sum_{j=m+\tau_{0}}^{\infty} \sum_{i=j}^{\infty} \frac{1}{a_{i, n+\sigma_{0}}} \\
\quad \times \sum_{t=n+\sigma_{0}}^{\infty}\left[f\left(i, t, x_{i-\tau_{1, i}, t-\sigma_{1, t}}, \ldots, x_{i-\tau_{k, i}, t-\sigma_{k, t}}\right)-c_{i, t}\right], \quad(m, n) \in \mathbb{Z}_{m_{1}, n_{1}}, \\
T_{L} x_{m_{1}, n_{1}}, \quad(m, n) \in \mathbb{Z}_{\alpha, \beta} \backslash \mathbb{Z}_{m_{1}, n_{1}}
\end{array}\right.
$$


for each $x=\left\{x_{m, n}\right\}_{(m, n) \in \mathbb{Z}_{\alpha, \beta}} \in A(N, M)$. It follows from (2.3), (2.4), (2.23), (2.24), and (2.26)(2.29) that for $x=\left\{x_{m, n}\right\}_{(m, n) \in \mathbb{Z}_{\alpha, \beta}}, y=\left\{y_{m, n}\right\}_{(m, n) \in \mathbb{Z}_{\alpha, \beta}} \in A(N, M)$ and $(m, n) \in \mathbb{Z}_{m_{1}, n_{1}}$ :

$$
\begin{aligned}
& \left|T_{L} x_{m, n}-T_{L} y_{m, n}\right|=\mid \frac{x_{m+\tau_{0}, n+\sigma_{0}}-y_{m+\tau_{0}, n+\sigma_{0}}}{b_{m+\tau_{0}, n+\sigma_{0}}}-\frac{1}{b_{m+\tau_{0}, n+\sigma_{0}}} \sum_{j=m+\tau_{0}}^{\infty} \sum_{i=j}^{\infty} \frac{1}{a_{i, n+\sigma_{0}}} \\
& \times \sum_{t=n+\sigma_{0}}^{\infty}\left[f\left(i, t, x_{i-\tau_{1, i}, t-\sigma_{1, t}}, \ldots, x_{i-\tau_{k, i}, t-\sigma_{k, t}}\right)\right. \\
& \left.-f\left(i, t, y_{i-\tau_{1, i}, t-\sigma_{1, t}}, \ldots, y_{i-\tau_{k, i}, t-\sigma_{k, t}}\right)\right] \mid \\
& \leq-\frac{\left|x_{m+\tau_{0}, n+\sigma_{0}}-y_{m+\tau_{0}, n+\sigma_{0}}\right|}{b_{m+\tau_{0}, n+\sigma_{0}}}-\frac{1}{b_{m+\tau_{0}, n+\sigma_{0}}} \sum_{j=m+\tau_{0}}^{\infty} \sum_{i=j}^{\infty} \frac{1}{\left|a_{i, n+\sigma_{0}}\right|} \\
& \times \sum_{t=n+\sigma_{0}}^{\infty} \mid f\left(i, t, x_{i-\tau_{1, i}, t-\sigma_{1, t}} \ldots, x_{i-\tau_{k, i}, t-\sigma_{k, t}}\right) \\
& -f\left(i, t, y_{i-\tau_{1, i}, t-\sigma_{1, t}} \ldots, y_{i-\tau_{k, i}, t-\sigma_{k, t}}\right) \mid \\
& \leq-\frac{\|x-y\|}{b_{1}}-\frac{1}{b_{1}} \sum_{j=m+\tau_{0}}^{\infty} \sum_{i=j}^{\infty} \frac{1}{\left|a_{i, n+\sigma_{0}}\right|} \\
& \times \sum_{t=n+\sigma_{0}}^{\infty} P_{i, t} \max \left\{\left|x_{i-\tau_{l, i}, t-\sigma_{l, t}}-y_{i-\tau_{l, i}, t-\sigma_{l, t}}\right|: 1 \leq l \leq k\right\} \\
& \leq-\frac{1}{b_{1}}\left(1+\sum_{j=m_{1}}^{\infty} \sum_{i=j}^{\infty} \sup _{n \in \mathbb{N}_{n_{1}}}\left\{\frac{1}{\left|a_{i, n}\right|} \sum_{t=n}^{\infty} P_{i, t}\right\}\right)\|x-y\|=\theta\|x-y\|, \\
& T_{L} x_{m, n}=\frac{L}{b_{m+\tau_{0}, n+\sigma_{0}}}-\frac{x_{m+\tau_{0}, n+\sigma_{0}}}{b_{m+\tau_{0}, n+\sigma_{0}}}+\frac{1}{b_{m+\tau_{0}, n+\sigma_{0}}} \sum_{j=m+\tau_{0}}^{\infty} \sum_{i=j}^{\infty} \frac{1}{a_{i, n+\sigma_{0}}} \\
& \times \sum_{t=n+\sigma_{0}}^{\infty}\left[f\left(i, t, x_{i-\tau_{1, i}, t-\sigma_{1, t}}, \ldots, x_{i-\tau_{k, i}, t-\sigma_{k, t}}\right)-c_{i, t}\right] \\
& \leq \frac{L}{b_{1}}-\frac{M}{b_{1}}-\frac{1}{b_{1}} \sum_{j=m+\tau_{0}}^{\infty} \sum_{i=j}^{\infty} \frac{1}{\left|a_{i, n+\sigma_{0}}\right|} \\
& \times \sum_{t=n+\sigma_{0}}^{\infty}\left[\left|f\left(i, t, x_{i-\tau_{1, i}, t-\sigma_{1, t}} \ldots, x_{i-\tau_{k, i}, t-\sigma_{k, t}}\right)\right|+\left|c_{i, t}\right|\right] \\
& \leq \frac{L}{b_{1}}-\frac{M}{b_{1}}-\frac{1}{b_{1}} \sum_{j=m+\tau_{0}}^{\infty} \sum_{i=j}^{\infty} \frac{1}{\left|a_{i, n+\sigma_{0}}\right|} \sum_{t=n+\sigma_{0}}^{\infty}\left(Q_{i, t}+\left|c_{i, t}\right|\right) \\
& \leq \frac{L}{b_{1}}-\frac{M}{b_{1}}-\frac{1}{b_{1}} \sum_{j=m_{1}}^{\infty} \sum_{i=j}^{\infty} \sup _{n \in \mathbb{N}_{n_{1}}}\left\{\frac{1}{\left|a_{i, n}\right|} \sum_{t=n}^{\infty}\left(Q_{i, t}+\left|c_{i, t}\right|\right)\right\} \\
& \leq \frac{L}{b_{1}}-\frac{M}{b_{1}}-\frac{1}{b_{1}} \min \left\{L-M\left(1+b_{1}\right), b_{1} N\left(1+\frac{1}{b_{2}}\right)-\frac{b_{1} L}{b_{2}}\right\} \leq M,
\end{aligned}
$$




$$
\begin{aligned}
T_{L} x_{m, n}= & \frac{L}{b_{m+\tau_{0}, n+\sigma_{0}}}-\frac{x_{m+\tau_{0}, n+\sigma_{0}}}{b_{m+\tau_{0}, n+\sigma_{0}}}+\frac{1}{b_{m+\tau_{0}, n+\sigma_{0}}} \sum_{j=m+\tau_{0}}^{\infty} \sum_{i=j}^{\infty} \frac{1}{a_{i, n+\sigma_{0}}} \\
& \times \sum_{t=n+\sigma_{0}}^{\infty}\left[f\left(i, t, x_{i-\tau_{1, i}, t-\sigma_{1, t}} \ldots, x_{i-\tau_{k, i}, t-\sigma_{k, t}}\right)-c_{i, t}\right] \\
\geq & \frac{L}{b_{2}}-\frac{N}{b_{2}}+\frac{1}{b_{1}} \sum_{j=m+\tau_{0}}^{\infty} \sum_{i=j}^{\infty} \frac{1}{\left|a_{i, n+\sigma_{0}}\right|} \\
& \times \sum_{t=n+\sigma_{0}}^{\infty}\left[\left|f\left(i, t, x_{i-\tau_{1, i}, t-\sigma_{1, t}} \ldots, x_{i-\tau_{k, i}, t-\sigma_{k, t}}\right)\right|+\left|c_{i, t}\right|\right] \\
\geq & \frac{L}{b_{2}}-\frac{N}{b_{2}}+\frac{1}{b_{1}} \sum_{j=m+\tau_{0}}^{\infty} \sum_{i=j}^{\infty} \frac{1}{\left|a_{i, n+\sigma_{0}}\right|} \sum_{t=n+\sigma_{0}}^{\infty}\left(Q_{i, t}+\left|c_{i, t}\right|\right) \\
\geq & \frac{L}{b_{2}}-\frac{N}{b_{2}}+\frac{1}{b_{1}} \sum_{j=m_{1}}^{\infty} \sum_{i=j}^{\infty} \sup _{n \in \mathbb{N}_{n_{1}}}\left\{\frac{1}{\left|a_{i, n}\right|} \sum_{t=n}^{\infty}\left(Q_{i, t}+\left|c_{i, t}\right|\right)\right\} \\
\geq & \frac{L}{b_{2}}-\frac{N}{b_{2}}+\frac{1}{b_{1}} \min \left\{L-M\left(1+b_{1}\right), b_{1} N\left(1+\frac{1}{b_{2}}\right)-\frac{b_{1} L}{b_{2}}\right\} \geq N,
\end{aligned}
$$

which imply that (2.15) holds. Consequently, (2.15) ensures that $T_{L}$ is a contraction mapping in $A(N, M)$ and it has a unique fixed-point $x=\left\{x_{m, n}\right\}_{(m, n) \in \mathbb{Z}_{\alpha, \beta}} \in A(N, M)$, which together with (2.29) gives that

$$
\begin{aligned}
x_{m, n}= & \frac{L}{b_{m+\tau_{0}, n+\sigma_{0}}}-\frac{x_{m+\tau_{0}, n+\sigma_{0}}}{b_{m+\tau_{0}, n+\sigma_{0}}}+\frac{1}{b_{m+\tau_{0}, n+\sigma_{0}}} \sum_{j=m+\tau_{0}}^{\infty} \sum_{i=j}^{\infty} \frac{1}{a_{i, n+\sigma_{0}}} \\
& \times \sum_{t=n+\sigma_{0}}^{\infty}\left[f\left(i, t, x_{i-\tau_{1, i}, t-\sigma_{1, t}}, \ldots, x_{i-\tau_{k, i}, t-\sigma_{k, t}}\right)-c_{i, t}\right], \quad(m, n) \in \mathbb{Z}_{m_{1}, n_{1}},
\end{aligned}
$$

which yields that for $(m, n) \in \mathbb{Z}_{m_{1}, n_{1}}$

$$
\begin{aligned}
\Delta_{m}\left(x_{m, n}+b_{m, n} x_{m-\tau_{0}, n-\sigma_{0}}\right) & =-\sum_{i=m}^{\infty} \frac{1}{a_{i, n}} \sum_{t=n}^{\infty}\left[f\left(i, t, x_{i-\tau_{1, i}, t-\sigma_{1, t}}, \ldots, x_{i-\tau_{k, i}, t-\sigma_{k, t}}\right)-c_{i, t}\right] \\
\Delta_{m}^{2}\left(x_{m, n}+b_{m, n} x_{m-\tau_{0}, n-\sigma_{0}}\right) & =\frac{1}{a_{m, n}} \sum_{t=n}^{\infty}\left[f\left(m, t, x_{m-\tau_{1, m}, t-\sigma_{1, t}}, \ldots, x_{m-\tau_{k, m}, t-\sigma_{k, t}}\right)-c_{m, t}\right] \\
\Delta_{n}\left(a_{m, n} \Delta_{m}^{2}\left(x_{m, n}+b_{m, n} x_{m-\tau_{0}, n-\sigma_{0}}\right)\right) & =-f\left(m, n, x_{m-\tau_{1, m}, n-\sigma_{1, t}} \ldots, x_{m-\tau_{k, m}, t-\sigma_{k, t}}\right)+c_{m, n},
\end{aligned}
$$

which implies that $x=\left\{x_{m, n}\right\}_{(m, n) \in \mathbb{Z}_{\alpha, \beta}}$ is a bounded positive solution of (1.11) in $A(N, M)$. 
It follows from (2.15), (2.25) and (2.29) that for any $s \in \mathbb{N}_{0}$ and $(m, n) \in \mathbb{Z}_{m_{1}, n_{1}}$

$$
\begin{aligned}
&\left|x_{m, n}(s+1)-x_{m, n}\right|=\mid[1-\alpha(s)-\beta(s)] x_{m, n}(s)+\alpha(s) \quad \times\left\{\frac{L}{b_{m+\tau_{0}, n+\sigma_{0}}}-\frac{x_{m+\tau_{0}, n+\sigma_{0}}(s)}{b_{m+\tau_{0}, n+\sigma_{0}}}+\frac{1}{b_{m+\tau_{0}, n+\sigma_{0}}}\right. \\
&\left.\quad \times \sum_{j=m+\tau_{0}}^{\infty} \sum_{i=j}^{\infty} \frac{1}{a_{i, n+\sigma_{0}}} \sum_{t=n+\sigma_{0}}^{\infty}\left[f\left(i, t, x_{i-\tau_{1, i}, t-\sigma_{1, t}}(s), \ldots, x_{i-\tau_{k, i}, t-\sigma_{k, t}}(s)\right)-c_{i, t}\right]\right\} \\
& \\
&+\beta(s) \gamma_{m, n}(s)-x_{m, n} \mid \\
& \leq[1-\alpha(s)-\beta(s)]\left|x_{m, n}(s)-x_{m, n}\right|+\alpha(s)\left|T_{L} x_{m, n}(s)-T_{L} x_{m, n}\right| \\
&+\beta(s)\left|\gamma_{m, n}(s)-x_{m, n}\right| \\
& \leq {[1-\alpha(s)-\beta(s)]\|x(s)-x\|+\alpha(s) \theta\|x(s)-x\|+2 M \beta(s) } \\
& \leq {[1-(1-\theta) \alpha(s)]\|x(s)-x\|+2 M \beta(s), }
\end{aligned}
$$

which yields (2.7). Thus Lemma 1.1 and (2.7)-(2.9) ensure that $\lim _{s \rightarrow \infty} x(s)=x$.

Next we show that (b) holds. Let $L_{1}, L_{2} \in\left(M\left(1+b_{1}\right), N\left(1+b_{2}\right)\right)$ let and $L_{1} \neq L_{2}$. As in the proof of (a), we infer that for each $i \in\{1,2\}$, there exist $\theta_{i}, m_{i+1}, n_{i+1}$ and $T_{L_{i}}$ satisfying (2.26)(2.29), where $\theta, m_{1}, n_{1}, L$ and $T_{L}$ are replaced by $\theta_{i}, m_{i+1}, n_{i+1}, L_{i}$ and $T_{L_{i}}$, respectively, and the mapping $T_{L_{i}}$ has a fixed-point $x^{i}=\left\{x_{m, n}^{i}\right\}_{(m, n) \in \mathbb{Z}_{\alpha, \beta}} \in A(N, M)$, which is a bounded positive solution of (1.11), that is:

$$
\begin{aligned}
x_{m, n}^{1}= & \frac{L_{1}}{b_{m+\tau_{0}, n+\sigma_{0}}}-\frac{x_{m+\tau_{0}, n+\sigma_{0}}^{1}}{b_{m+\tau_{0}, n+\sigma_{0}}}+\frac{1}{b_{m+\tau_{0}, n+\sigma_{0}}} \sum_{j=m+\tau_{0}}^{\infty} \sum_{i=j}^{\infty} \frac{1}{a_{i, n+\sigma_{0}}} \\
& \times \sum_{t=n+\sigma_{0}}^{\infty}\left[f\left(i, t, x_{i-\tau_{1, i}, t-\sigma_{1, t}}^{1} \ldots, x_{i-\tau_{k, i}, t-\sigma_{k, t}}^{1}\right)-c_{i, t}\right], \quad(m, n) \in \mathbb{Z}_{m_{2}, n_{2},} \\
x_{m, n}^{2}= & \frac{L_{2}}{b_{m+\tau_{0}, n+\sigma_{0}}}-\frac{x_{m+\tau_{0}, n+\sigma_{0}}^{2}}{b_{m+\tau_{0}, n+\sigma_{0}}}+\frac{1}{b_{m+\tau_{0}, n+\sigma_{0}}} \sum_{j=m+\tau_{0}}^{\infty} \sum_{i=j}^{\infty} \frac{1}{a_{i, n+\sigma_{0}}} \\
& \times \sum_{t=n+\sigma_{0}}^{\infty}\left[f\left(i, t, x_{i-\tau_{1, i}, t-\sigma_{1, t}}^{2}, \ldots, x_{i-\tau_{k, i}, t-\sigma_{k, t}}^{2}\right)-c_{i, t}\right], \quad(m, n) \in \mathbb{Z}_{m_{3}, n_{3} .} .
\end{aligned}
$$


In order to show that the set of bounded positive solutions of (1.11) is uncountable, it is sufficient to prove that $x^{1} \neq x^{2}$. It follows from (2.3), (2.26), (2.27), (2.34), and (2.35) that for $(m, n) \in \mathbb{Z}_{\max \left\{m_{2}, m_{3}\right\}, \max \left\{n_{2}, n_{3}\right\}}$

$$
\begin{aligned}
& \left|x_{m, n}^{1}-x_{m, n}^{2}\right|=\mid \frac{L_{1}-L_{2}}{b_{m+\tau_{0}, n+\sigma_{0}}}-\frac{x_{m+\tau_{0}, n+\sigma_{0}}^{1}-x_{m+\tau_{0}, n+\sigma_{0}}^{2}}{b_{m+\tau_{0}, n+\sigma_{0}}}+\frac{1}{b_{m+\tau_{0}, n+\sigma_{0}}} \sum_{j=m+\tau_{0}}^{\infty} \sum_{i=j}^{\infty} \frac{1}{a_{i, n+\sigma_{0}}} \\
& \times \sum_{t=n+\sigma_{0}}^{\infty}\left[f\left(i, t, x_{i-\tau_{1, i}, t-\sigma_{1, t}}^{1} \ldots, x_{i-\tau_{k, i}, t-\sigma_{k, t}}^{1}\right)-f\left(i, t, x_{i-\tau_{1, i}, t-\sigma_{1, t}}^{2} \ldots, x_{i-\tau_{k, i}, t-\sigma_{k, t}}^{2}\right)\right] \mid \\
& \geq-\frac{\left|L_{1}-L_{2}\right|}{b_{m+\tau_{0}, n+\sigma_{0}}}+\frac{\left|x_{m+\tau_{0}, n+\sigma_{0}}^{1}-x_{m+\tau_{0}, n+\sigma_{0}}^{2}\right|}{b_{m+\tau_{0}, n+\sigma_{0}}}+\frac{1}{b_{m+\tau_{0}, n+\sigma_{0}}} \sum_{j=m+\tau_{0}}^{\infty} \sum_{i=j}^{\infty} \frac{1}{\left|a_{i, n+\sigma_{0}}\right|} \\
& \times \sum_{t=n+\sigma_{0}}^{\infty}\left|f\left(i, t, x_{i-\tau_{1, i}, t-\sigma_{1, t}}^{1} \ldots, x_{i-\tau_{k, i}, t-\sigma_{k, t}}^{1}\right)-f\left(i, t, x_{i-\tau_{1, i}, t-\sigma_{1, t^{\prime}}}^{2} \ldots, x_{i-\tau_{k, i}, t-\sigma_{k, t}}^{2}\right)\right| \\
& \geq-\frac{\left|L_{1}-L_{2}\right|}{b_{2}}+\frac{\left\|x^{1}-x^{2}\right\|}{b_{1}}+\frac{1}{b_{1}} \sum_{j=m+\tau_{0}}^{\infty} \sum_{i=j}^{\infty} \frac{1}{\left|a_{i, n+\sigma_{0}}\right|} \\
& \times \sum_{t=n+\sigma_{0}}^{\infty} P_{i, t} \max \left\{\left|x_{i-\tau_{1, i}, t-\sigma_{1, t}}^{1}-x_{i-\tau_{1, i}, t-\sigma_{1, t}}^{2}\right|: 1 \leq l \leq k\right\} \\
& \geq-\frac{\left|L_{1}-L_{2}\right|}{b_{2}}+\frac{1}{b_{1}}\left(1+\sum_{j=m+\tau_{0}}^{\infty} \sum_{i=j}^{\infty} \frac{1}{\left|a_{i, n+\sigma_{0}}\right|} \sum_{t=n+\sigma_{0}}^{\infty} P_{i, t}\right)\left\|x^{1}-x^{2}\right\|
\end{aligned}
$$

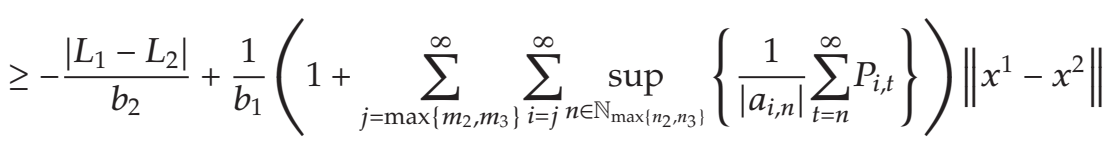

$$
\begin{aligned}
& \geq-\frac{\left|L_{1}-L_{2}\right|}{b_{2}}-\max \left\{\theta_{1}, \theta_{2}\right\}\left\|x^{1}-x^{2}\right\|
\end{aligned}
$$

which implies that

$$
\left\|x^{1}-x^{2}\right\| \geq-\frac{\left|L_{1}-L_{2}\right|}{b_{2}\left(1+\max \left\{\theta_{1}, \theta_{2}\right\}\right)}>0
$$

that is, $x^{1} \neq x^{2}$. This completes the proof.

Theorem 2.3. Assume that there exist positive constants $M$ and $N$, nonnegative constants $b_{1}$ and $b_{2}$, and nonnegative sequences $\left\{P_{m, n}\right\}_{(m, n) \in \mathbb{N}_{m_{0}, n_{0}}}$ and $\left\{Q_{m, n}\right\}_{(m, n) \in \mathbb{N}_{m_{0}, n_{0}}}$ satisfying (2.3)-(2.5), (2.24) and

$$
1<b_{2}, \quad b_{1}<b_{2}^{2}, \quad M b_{1}\left(b_{2}^{2}-b_{1}\right)>N b_{2}\left(b_{1}^{2}-b_{2}\right) .
$$


Then

(a) for any $L \in\left(b_{1} N+b_{1} M / b_{2}, b_{2} M+b_{2} N / b_{1}\right)$, there exist $\theta \in(0,1), m_{1} \geq m_{0}+\tau_{0}+|\alpha|$ and $n_{1} \geq n_{0}+\sigma_{0}+|\beta|$ such that for each $x(0)=\left\{x_{m, n}(0)\right\}_{(m, n) \in \mathbb{Z}_{\alpha, \beta}} \in A(N, M)$, the Mann iterative sequence with errors $\{x(s)\}_{s \in \mathbb{N}_{0}}=\left\{x_{m, n}(s)\right\}_{(m, n, s) \in \mathbb{Z}_{\alpha, \beta} \times \mathbb{N}_{0}}$ generated by (2.25) converges to a bounded positive solution $x \in A(N, M)$ of (1.11) and has the error estimate (2.7), where $\{\gamma(s)\}_{s \geq 0}$ is an arbitrary sequence in $A(N, M),\{\alpha(s)\}_{s \geq 0}$ and $\{\beta(s)\}_{s \geq 0}$ are any sequences in $[0,1]$ satisfying $(2.8)$ and (2.9);

(b) (1.11) possesses uncountably many bounded positive solutions in $A(M, N)$.

Proof. Set $\left.L \in\left(b_{1} N+b_{1} M / b_{2}\right), b_{2} M+b_{2} N / b_{1}\right)$. It follows from (2.5), (2.24), and (2.38) that there exist $\theta \in(0,1), m_{1} \geq m_{0}+\tau_{0}+|\alpha|$ and $n_{1} \geq n_{0}+\sigma_{0}+|\beta|$ satisfying (2.27):

$$
\begin{gathered}
\theta=\frac{1}{b_{2}}\left(1+\sum_{j=m_{1}}^{\infty} \sum_{i=j}^{\infty} \sup _{n \in \mathbb{N}_{n_{1}}}\left\{\frac{1}{\left|a_{i, n}\right|} \sum_{t=n}^{\infty} P_{i, t}\right\}\right), \\
\sum_{j=m_{1}}^{\infty} \sum_{i=j}^{\infty} \sup _{n \in \mathbb{N}_{n_{1}}}\left\{\frac{1}{\left|a_{i, n}\right|} \sum_{t=n}^{\infty}\left(Q_{i, t}+\left|c_{i, t}\right|\right)\right\} \leq \min \left\{b_{2} M-L+\frac{b_{2} N}{b_{1}}, \frac{b_{2} L}{b_{1}}-M-b_{2} N\right\} .
\end{gathered}
$$

Let the mapping $T_{L}: A(N, M) \rightarrow l_{\alpha, \beta}^{\infty}$ be defined by (2.29). It follows from (2.3), (2.4), (2.24), (2.27), (2.29), and (2.38)-(2.40) that for $x=\left\{x_{m, n}\right\}_{(m, n) \in \mathbb{Z}_{\alpha, \beta}} y=\left\{y_{m, n}\right\}_{(m, n) \in \mathbb{Z}_{\alpha, \beta}} \in A(N, M)$ and $(m, n) \in \mathbb{Z}_{m_{1}, n_{1}}$

$$
\begin{aligned}
& \left|T_{L} x_{m, n}-T_{L} y_{m, n}\right|=\mid \frac{x_{m+\tau_{0}, n+\sigma_{0}}-y_{m+\tau_{0}, n+\sigma_{0}}}{b_{m+\tau_{0}, n+\sigma_{0}}}-\frac{1}{b_{m+\tau_{0}, n+\sigma_{0}}} \sum_{j=m+\tau_{0}}^{\infty} \sum_{i=j}^{\infty} \frac{1}{a_{i, n+\sigma_{0}}} \\
& \times \sum_{t=n+\sigma_{0}}^{\infty}\left[f\left(i, t, x_{i-\tau_{1, i}, t-\sigma_{1, t}}, \ldots, x_{i-\tau_{k, i}, t-\sigma_{k, t}}\right)\right. \\
& \left.-f\left(i, t, y_{i-\tau_{1, i}, t-\sigma_{1, t}}, \ldots, y_{i-\tau_{k, i}, t-\sigma_{k, t}}\right)\right] \mid \\
& \leq \frac{\left|x_{m+\tau_{0}, n+\sigma_{0}}-y_{m+\tau_{0}, n+\sigma_{0}}\right|}{b_{m+\tau_{0}, n+\sigma_{0}}}+\frac{1}{b_{m+\tau_{0}, n+\sigma_{0}}} \sum_{j=m+\tau_{0}}^{\infty} \sum_{i=j}^{\infty} \frac{1}{\left|a_{i, n+\sigma_{0}}\right|} \\
& \times \sum_{t=n+\sigma_{0}}^{\infty} \mid f\left(i, t, x_{i-\tau_{1, i}, t-\sigma_{1, t}}, \ldots, x_{i-\tau_{k, i}, t-\sigma_{k, t}}\right) \\
& -f\left(i, t, y_{i-\tau_{1, i}, t-\sigma_{1, t}}, \ldots, y_{i-\tau_{k, i}, t-\sigma_{k, t}}\right) \mid \\
& \leq \frac{\|x-y\|}{b_{2}}+\frac{1}{b_{2}} \sum_{j=m+\tau_{0}}^{\infty} \sum_{i=j}^{\infty} \frac{1}{\left|a_{i, n+\sigma_{0}}\right|} \\
& \times \sum_{t=n+\sigma_{0}}^{\infty} P_{i, t} \max \left\{\left|x_{i-\tau_{l, i}, t-\sigma_{l, t}}-y_{i-\tau_{l, i}, t-\sigma_{l, t}}\right|: 1 \leq l \leq k\right\} \\
& \leq \frac{1}{b_{2}}\left(1+\sum_{j=m_{1}}^{\infty} \sum_{i=j}^{\infty} \sup _{n \in \mathbb{N}_{n_{1}}}\left\{\frac{1}{\left|a_{i, n}\right|} \sum_{t=n}^{\infty} P_{i, t}\right\}\right)\|x-y\|=\theta\|x-y\|,
\end{aligned}
$$




$$
\begin{aligned}
& T_{L} x_{m, n}=\frac{L}{b_{m+\tau_{0}, n+\sigma_{0}}}-\frac{x_{m+\tau_{0}, n+\sigma_{0}}}{b_{m+\tau_{0}, n+\sigma_{0}}}+\frac{1}{b_{m+\tau_{0}, n+\sigma_{0}}} \sum_{j=m+\tau_{0, m}}^{\infty} \sum_{i=j}^{\infty} \frac{1}{a_{i, n+\sigma_{0}}} \\
& \times \sum_{t=n+\sigma_{0}}^{\infty}\left[f\left(i, t, x_{i-\tau_{1, i}, t-\sigma_{1, t}}, \ldots, x_{i-\tau_{k, i}, t-\sigma_{k, t}}\right)-c_{i, t}\right] \\
& \leq \frac{L}{b_{2}}-\frac{N}{b_{1}}+\frac{1}{b_{2}} \sum_{j=m+\tau_{0}}^{\infty} \sum_{i=j}^{\infty} \frac{1}{\left|a_{i, n+\sigma_{0}}\right|} \\
& \times \sum_{t=n+\sigma_{0}}^{\infty}\left[\left|f\left(i, t, x_{i-\tau_{1, i}, t-\sigma_{1, t}} \ldots, x_{i-\tau_{k, i}, t-\sigma_{k, t}}\right)\right|+\left|c_{i, t}\right|\right] \\
& \leq \frac{L}{b_{2}}-\frac{N}{b_{1}}+\frac{1}{b_{2}} \sum_{j=m_{1}}^{\infty} \sum_{i=j}^{\infty} \sup _{n \in \mathbb{N}_{n_{1}}}\left\{\frac{1}{\left|a_{i, n}\right|} \sum_{t=n}^{\infty}\left(Q_{i, t}+\left|c_{i, t}\right|\right)\right\} \\
& \leq \frac{L}{b_{2}}-\frac{N}{b_{1}}+\frac{1}{b_{2}} \min \left\{b_{2} M-L+\frac{b_{2} N}{b_{1}}, \frac{b_{2} L}{b_{1}}-M-b_{2} N\right\} \leq M, \\
& T_{L} x_{m, n}=\frac{L}{b_{m+\tau_{0}, n+\sigma_{0}}}-\frac{x_{m+\tau_{0}, n+\sigma_{0}}}{b_{m+\tau_{0}, n+\sigma_{0}}}+\frac{1}{b_{m+\tau_{0}, n+\sigma_{0}}} \sum_{j=m+\tau_{0, m}}^{\infty} \sum_{i=j}^{\infty} \frac{1}{a_{i, n+\sigma_{0}}} \\
& \times \sum_{t=n+\sigma_{0}}^{\infty}\left[f\left(i, t, x_{i-\tau_{1, i}, t-\sigma_{1, t}}, \ldots, x_{i-\tau_{k, i}, t-\sigma_{k, t}}\right)-c_{i, t}\right] \\
& \geq \frac{L}{b_{1}}-\frac{M}{b_{2}}-\frac{1}{b_{2}} \sum_{j=m+\tau_{0}}^{\infty} \sum_{i=j}^{\infty} \frac{1}{\left|a_{i, n+\sigma_{0}}\right|} \\
& \times \sum_{t=n+\sigma_{0}}^{\infty}\left[\left|f\left(i, t, x_{i-\tau_{1, i}, t-\sigma_{1, t}}, \ldots, x_{i-\tau_{k, i}, t-\sigma_{k, t}}\right)\right|+\left|c_{i, t}\right|\right] \\
& \geq \frac{L}{b_{1}}-\frac{M}{b_{2}}-\frac{1}{b_{2}} \sum_{j=m_{1}}^{\infty} \sum_{i=j}^{\infty} \sup _{n \in \mathbb{N}_{n_{1}}}\left\{\frac{1}{a_{i, n}} \sum_{t=n}^{\infty}\left(Q_{i, t}+\left|c_{i, t}\right|\right)\right\} \\
& \geq \frac{L}{b_{1}}-\frac{M}{b_{2}}-\frac{1}{b_{2}} \min \left\{b_{2} M-L+\frac{b_{2} N}{b_{1}}, \frac{b_{2} L}{b_{1}}-M-b_{2} N\right\} \geq N \text {, }
\end{aligned}
$$

which imply that (2.15) holds. Consequently (2.15) ensures that $T_{L}$ is a contraction mapping, and hence it has a unique fixed-point $x=\left\{x_{m, n}\right\}_{(m, n) \in \mathbb{Z}_{\alpha, \beta}} \in A(N, M)$, which gives that

$$
\begin{aligned}
x_{m, n}= & \frac{L}{b_{m+\tau_{0}, n+\sigma_{0}}}-\frac{x_{m+\tau_{0}, n+\sigma_{0}}}{b_{m+\tau_{0}, n+\sigma_{0}}}+\frac{1}{b_{m+\tau_{0}, n+\sigma_{0}}} \sum_{j=m+\tau_{0}}^{\infty} \sum_{i=j}^{\infty} \frac{1}{a_{i, n+\sigma_{0}}} \\
& \times \sum_{t=n+\sigma_{0}}^{\infty}\left[f\left(i, t, x_{i-\tau_{1, i}, t-\sigma_{1, t}} \ldots, x_{i-\tau_{k, i}, t-\sigma_{k, t}}\right)-c_{i, t}\right],(m, n) \in \mathbb{Z}_{m_{1}, n_{1}} .
\end{aligned}
$$

As in the proof of Theorem 2.2, it is easy to verify that $x=\left\{x_{m, n}\right\}_{(m, n) \in \mathbb{Z}_{\alpha, \beta}}$ is a bounded positive solution of $(1.11)$ in $A(N, M)$; (2.7) holds and $\lim _{s \rightarrow \infty} x(s)=x$. 
Next we show that (b) holds. Let $L_{1}, L_{2} \in\left(b_{1} N+b_{1} M / b_{2}, b_{2} M+b_{2} N / b_{1}\right)$ and $L_{1} \neq L_{2}$. As in the proof of (a), we infer that for each $i \in\{1,2\}$, there exist $\theta_{i}, m_{i+1}, n_{i+1}$ and $T_{L_{i}}$ satisfying (2.27), (2.29), (2.39), and (2.40), where $\theta, m_{1}, n_{1}, L$ and $T_{L}$ are replaced by $\theta_{i}, m_{i+1}, n_{i+1}, L_{i}$, and $T_{L_{i}}$, respectively, and the mapping $T_{L_{i}}$ has a fixed-point $x^{i}=\left\{x_{m, n}^{i}\right\}_{(m, n) \in \mathbb{Z}_{\alpha, \beta}} \in A(N, M)$, which is a bounded positive solution of (1.11) and satisfies (2.34) and (2.35). In order to show that the set of bounded positive solutions of (1.11) is uncountable, it is sufficient to prove that $x^{1} \neq x^{2}$. It follows from (2.3), (2.27), (2.34), (2.35), and (2.39) that for $(m, n) \in$ $\mathbb{Z}_{\max \left\{m_{2}, m_{3}\right\}, \max \left\{n_{2}, n_{3}\right\}}$

$$
\begin{aligned}
& \left|x_{m, n}^{1}-x_{m, n}^{2}\right|=\mid \frac{L_{1}-L_{2}}{b_{m+\tau_{0}, n+\sigma_{0}}}-\frac{x_{m+\tau_{0}, n+\sigma_{0}}^{1}-x_{m+\tau_{0}, n+\sigma_{0}}^{2}}{b_{m+\tau_{0}, n+\sigma_{0}}}+\frac{1}{b_{m+\tau_{0}, n+\sigma_{0}}} \sum_{j=m+\tau_{0}}^{\infty} \sum_{i=j}^{\infty} \frac{1}{a_{i, n+\sigma_{0}}} \\
& \times \sum_{t=n+\sigma_{0}}^{\infty}\left[f\left(i, t, x_{i-\tau_{1, i}, t-\sigma_{1, t}}^{1} \ldots, x_{i-\tau_{k, i}, t-\sigma_{k, t}}^{1}\right)\right. \\
& \left.-f\left(i, t, x_{i-\tau_{1, i}, t-\sigma_{1, t}}^{2}, \ldots, x_{i-\tau_{k, i}, t-\sigma_{k, t}}^{2}\right)\right] \mid \\
& \geq \frac{\left|L_{1}-L_{2}\right|}{b_{m+\tau_{0}, n+\sigma_{0}}}-\frac{\left|x_{m+\tau_{0}, n+\sigma_{0}}^{1}-x_{m+\tau_{0}, n+\sigma_{0}}^{2}\right|}{b_{m+\tau_{0}, n+\sigma_{0}}}-\frac{1}{b_{m+\tau_{0}, n+\sigma_{0}}} \sum_{j=m+\tau_{0}}^{\infty} \sum_{i=j}^{\infty} \frac{1}{\left|a_{i, n+\sigma_{0}}\right|} \\
& \times \sum_{t=n+\sigma_{0}}^{\infty}\left|f\left(i, t, x_{i-\tau_{1, i}, t-\sigma_{1, t}}^{1} \ldots, x_{i-\tau_{k, i}, t-\sigma_{k, t}}^{1}\right)-f\left(i, t, x_{i-\tau_{1, i}, t-\sigma_{1, t}}^{2}, \ldots, x_{i-\tau_{k, i}, t-\sigma_{k, t}}^{2}\right)\right| \\
& \geq \frac{\left|L_{1}-L_{2}\right|}{b_{1}}-\frac{\left\|x^{1}-x^{2}\right\|}{b_{2}}-\frac{1}{b_{2}} \sum_{j=m+\tau_{0}}^{\infty} \sum_{i=j}^{\infty} \frac{1}{\left|a_{i, n+\sigma_{0}}\right|} \\
& \times \sum_{t=n+\sigma_{0}}^{\infty} P_{i, t} \max \left\{\left|x_{i-\tau_{1, i}, t-\sigma_{1, t}}^{1}-x_{i-\tau_{1, i}, t-\sigma_{1, t}}^{2}\right|: 1 \leq l \leq k\right\} \\
& \geq \frac{\left|L_{1}-L_{2}\right|}{b_{1}}-\frac{1}{b_{2}}\left(1+\sum_{j=m+\tau_{0}}^{\infty} \sum_{i=j}^{\infty} \frac{1}{\left|a_{i, n+\sigma_{0}}\right|} \sum_{t=n+\sigma_{0}}^{\infty} P_{i, t}\right)\left\|x^{1}-x^{2}\right\| \\
& \geq \frac{\left|L_{1}-L_{2}\right|}{b_{1}}-\frac{1}{b_{2}}\left(1+\sum_{j=\max \left\{m_{2}, m_{3}\right\}}^{\infty} \sum_{i=j}^{\infty} \sup _{n \in \mathbb{N}_{\left.\max \mid n_{2}, n_{3}\right\}}}\left\{\frac{1}{\left|a_{i, n}\right|} \sum_{t=n}^{\infty} P_{i, t}\right\}\right)\left\|x^{1}-x^{2}\right\| \\
& \geq \frac{\left|L_{1}-L_{2}\right|}{b_{1}}-\max \left\{\theta_{1}, \theta_{2}\right\}\left\|x^{1}-x^{2}\right\|
\end{aligned}
$$

which implies that

$$
\left\|x^{1}-x^{2}\right\| \geq \frac{\left|L_{1}-L_{2}\right|}{b_{1}\left(1+\max \left\{\theta_{1}, \theta_{2}\right\}\right)}>0
$$

that is, $x^{1} \neq x^{2}$. This completes the proof. 


\section{Examples}

Now we illustrate the results presented in Section 2 with the following three examples. Note that none of the known results can be applied to the examples.

Example 3.1. Consider the third-order nonlinear partial difference equation with delays:

$$
\begin{gathered}
\Delta_{n}\left((-1)^{m n} m^{4} n^{3} \Delta_{m}^{2}\left(x_{m, n}+\frac{(-1)^{m+n}}{3} x_{m-\tau_{0}, n-\sigma_{0}}\right)\right)+\frac{\sqrt{n}}{m^{2}\left(n^{2}+1\right)} x_{m^{2}, n(n+1) / 2}^{3} \\
-\frac{\cos \left(m^{3} n^{5}-\ln m\right)}{(m+1)^{2} n^{2}} x_{m^{3}-2 m, n^{2}-n}^{2}=\frac{(-1)^{n} \sin \left(m^{2}-2 n\right)}{\sqrt{m^{5} n^{4}+1}}, \quad m \geq 1, n \geq 1,
\end{gathered}
$$

where $\tau_{0}, \sigma_{0} \in \mathbb{N}$ are fixed. Let $m_{0}=n_{0}=1, k=2, b_{1}=b_{2}=1 / 3, \alpha=\min \left\{1-\tau_{0},-1\right\}, \beta=1-\sigma_{0}$, $M$ and let $N$ be two positive constants with $M>3 N$ and

$$
\begin{gathered}
a_{m, n}=(-1)^{m n} m^{4} n^{3}, \quad b_{m, n}=\frac{(-1)^{m+n}}{3}, \quad c_{m, n}=\frac{(-1)^{m+n} \sin \left(m^{2}-2 n\right)}{\sqrt{m^{5} n^{4}+1}}, \\
f(m, n, u, v)=\frac{\sqrt{n}}{m^{2}\left(n^{2}+1\right)} u^{3}-\frac{\cos \left(m^{3} n^{5}-\ln m\right)}{(m+1)^{2} n^{2}} v^{2}, \\
\tau_{1, m}=m(1-m), \quad \tau_{2, m}=m\left(3-m^{2}\right), \quad \sigma_{1, n}=\frac{n(1-n)}{2}, \quad \sigma_{2, n}=n(2-n), \\
P_{m, n}=\frac{3 M^{2} \sqrt{n}}{m^{2}\left(n^{2}+1\right)}+\frac{2 M}{(m+1)^{2} n^{2}}, \quad Q_{m, n}=\frac{M^{3} \sqrt{n}}{m^{2}\left(n^{2}+1\right)}+\frac{M^{2}}{(m+1)^{2} n^{2}}, \\
(m, n, u, v) \in \mathbb{N}_{m_{0}, n_{0}} \times \mathbb{R}^{2} .
\end{gathered}
$$

It is easy to verify that (2.1)-(2.4) hold. Note that

$$
\begin{aligned}
\sum_{j=m_{0}}^{\infty} & \sum_{i=j}^{\infty} \sup _{n \geq n_{0}}\left\{\frac{1}{\left|a_{i, n}\right|} \sum_{t=n}^{\infty} \max \left\{P_{i, t}, Q_{i, t},\left|c_{i, t}\right|\right\}\right\} \\
& =\sum_{j=m_{0}}^{\infty} \sum_{i=j}^{\infty} \sup _{n \geq n_{0}}\left\{\frac{1}{i^{4} n^{3}} \sum_{t=n}^{\infty} \max \left\{\frac{3 M^{2} \sqrt{t}}{i^{2}\left(t^{2}+1\right)}+\frac{2 M}{(i+1)^{2} t^{2}}, \frac{M^{3} \sqrt{t}}{i^{2}\left(t^{2}+1\right)}+\frac{M^{2}}{(i+1)^{2} t^{2}}, \frac{\left|\sin \left(i^{2}-2 t\right)\right|}{\sqrt{i^{5} t^{4}+1}}\right\}\right\} \\
& <\left(1+2 M+4 M^{2}+M^{3}\right)\left(\sum_{t=n_{0}}^{\infty} \frac{1}{\sqrt{t^{3}}}\right) \sum_{j=m_{0}}^{\infty} \sum_{i=j}^{\infty} \frac{1}{i^{4}}<+\infty .
\end{aligned}
$$

Hence the conditions of Theorem 2.1 are fulfilled. It follows from Theorem 2.1 that (3.1) possesses uncountably many bounded positive solutions in $A(N, M)$. On the other hand, for any $L \in(N+(1 / 3) M,(2 / 3) M)$, there exist $\theta \in(0,1)$ and $m_{1} \geq m_{0}+\tau_{0}+|\alpha|, n_{1} \geq n_{0}+\sigma_{0}+|\beta|$ such that the Mann iterative sequence with errors $\{x(s)\}_{s \geq 0}$ generated by (2.6) converges to a bounded positive solution $x \in A(N, M)$ of (3.1) and has the error estimate (2.7), where $\{\gamma(s)\}_{s \geq 0}$ is an arbitrary sequence in $A(N, M),\{\alpha(s)\}_{s \geq 0}$ and $\{\beta(s)\}_{s \geq 0}$ are any sequences in $[0,1]$ satisfying $(2.8)$ and $(2.9)$. 
Example 3.2. Consider the third-order nonlinear partial difference equation with delays:

$$
\begin{gathered}
\Delta_{n}\left((-1)^{n} m^{3} \ln ^{2}(m+n) \Delta_{m}^{2}\left(x_{m, n}-\frac{4 n+(-1)^{m} n}{n+1} x_{m-\tau_{0}, n-\sigma_{0}}\right)\right) \\
+\frac{x_{m-2, n-3}^{2} x_{3 m^{2}-2, n^{5}-1}^{3}}{m^{3} n^{2}}=\frac{\cos \left(n m^{3}-\sqrt{m}\right)}{\sqrt{n^{3}+1}}, \quad m \geq 1, n \geq 1,
\end{gathered}
$$

where $\tau_{0}, \sigma_{0} \in \mathbb{N}$ are fixed. Let $m_{0}=n_{0}=1, k=2, b_{1}=-2, b_{2}=-5, \alpha=\min \left\{1-\tau_{0},-1\right\}, \beta=$ $\min \left\{1-\sigma_{0},-2\right\}, M$ and let $N$ be two positive constants with $M>4 N$ and

$$
\begin{gathered}
a_{m, n}=(-1)^{n} m^{3} \ln ^{2}(m+n), \quad b_{m, n}=-\frac{4 n+(-1)^{m} n}{n+1}, \quad c_{m, n}=\frac{\cos \left(n m^{3}-\sqrt{m}\right)}{\sqrt{n^{3}+1}}, \\
f(m, n, u, v)=\frac{u^{2} v^{3}}{m^{3} n^{2}}, \quad \tau_{1, m}=2, \quad \tau_{2, m}=-3 m^{2}+m+2, \quad \sigma_{1, n}=3, \quad \sigma_{2, n}=-n^{5}+n+1, \\
P_{m, n}=\frac{5 M^{4}}{m^{3} n^{2}}, \quad Q_{m, n}=\frac{M^{5}}{m^{3} n^{2}}, \quad(m, n, u, v) \in \mathbb{N}_{m_{0}, n_{0}} \times \mathbb{R}^{2} .
\end{gathered}
$$

It is clear that (2.3), (2.4), (2.23), and (2.24) hold. Observe that

$$
\begin{aligned}
\sum_{j=m_{0}}^{\infty} \sum_{i=j}^{\infty} \sup _{n \geq n_{0}}\left\{\frac{1}{\left|a_{i, n}\right|} \sum_{t=n}^{\infty} \max \left\{P_{i, t}, Q_{i, t},\left|c_{i, t}\right|\right\}\right\} \\
\quad=\sum_{j=m_{0}}^{\infty} \sum_{i=j}^{\infty} \sup _{n \geq n_{0}}\left\{\frac{1}{i^{3} \ln ^{2}(i+n)} \sum_{t=n}^{\infty} \max \left\{\frac{5 M^{4}}{i^{3} t^{2}}, \frac{M^{5}}{i^{3} t^{2}}, \frac{\left|\cos \left(t i^{3}-\sqrt{i}\right)\right|}{\sqrt{t^{3}+1}}\right\}\right\} \\
<\frac{1+5 M^{4}+M^{5}}{\ln ^{2} 2}\left(\sum_{t=n_{0}}^{\infty} \frac{1}{\sqrt{t^{3}}}\right) \sum_{j=m_{0}}^{\infty} \sum_{i=j}^{\infty} \frac{1}{i^{3}}<+\infty .
\end{aligned}
$$

That is, the conditions of Theorem 2.2 are fulfilled. Thus Theorem 2.2 ensures that (3.4) has uncountably many bounded positive solutions in $A(N, M)$. On the other hand, for any $L \in$ $(-M,-4 N)$, there exist $\theta \in(0,1)$ and $m_{1} \geq m_{0}+\tau_{0}+|\alpha|, n_{1} \geq n_{0}+\sigma_{0}+|\beta|$ such that the Mann iterative sequence with errors $\{x(s)\}_{s \geq 0}$ generated by (2.25) converges to a bounded positive solution $x \in A(N, M)$ of (3.4) and has the error estimate (2.7), where $\{\gamma(s)\}_{s \geq 0}$ is an arbitrary sequence in $A(N, M),\{\alpha(s)\}_{s \geq 0}$ and $\{\beta(s)\}_{s \geq 0}$ are any sequences in $[0,1]$ satisfying $(2.8)$ and (2.9). 
Example 3.3. Consider the third-order nonlinear partial difference equation with delays:

$$
\begin{aligned}
& \Delta_{n}\left((-1)^{n}\left(m^{5} n\right) \Delta_{m}^{2}\left(x_{m, n}+\frac{2 m n+3}{m n+1} x_{m-\tau_{0}, n-\sigma_{0}}\right)\right)+\frac{\left((-1)^{m}-1 / n\right) x_{m-4, n-3}^{4}}{m^{3} n^{2}+x_{m^{2}-2 m, n^{3}-n}^{2}} \\
& \quad=\frac{(-1)^{n+m}\left(m^{2}-3 n^{3}\right)}{m^{5}\left(n^{7}+1\right)}, \quad m \geq 1, n \geq 1,
\end{aligned}
$$

where $\tau_{0}, \sigma_{0} \in \mathbb{N}$ are fixed. Let $m_{0}=n_{0}=1, k=2, b_{1}=3, b_{2}=2, \alpha=\min \left\{1-\tau_{0},-3\right\}, \beta=$ $\min \left\{1-\sigma_{0},-2\right\}, M$ and $N$ be two positive constants with $M>(14 / 3) N$ and

$$
\begin{gathered}
a_{m, n}=(-1)^{n}\left(m^{5} n\right), \quad b_{m, n}=\frac{2 m n+3}{m n+1}, \quad c_{m, n}=\frac{(-1)^{n+m}\left(m^{2}-3 n^{3}\right)}{m^{5}\left(n^{7}+1\right)}, \\
f(m, n, u, v)=\frac{\left((-1)^{m}-1 / n\right) u^{4}}{m^{3} n^{2}+v^{2}}, \quad \tau_{1, m}=4, \quad \tau_{2, m}=m(3-m), \quad \sigma_{1, n}=3, \quad \sigma_{2, n}=-n^{3}+2 n, \\
P_{m, n}=\frac{2 M^{3}\left(2 m^{3} n^{2}+2 M^{2}+1\right)}{\left(m^{3} n^{2}+N^{2}\right)^{2}}, \quad Q_{m, n}=\frac{2 M^{4}}{m^{3} n^{2}+N^{2}}, \quad(m, n, u, v) \in \mathbb{N}_{m_{0}, n_{0}} \times \mathbb{R}^{2} .
\end{gathered}
$$

Clearly (2.3), (2.4), (2.24), and (2.38) hold. Notice that

$$
\begin{aligned}
\sum_{j=m_{0}}^{\infty} & \sum_{i=j}^{\infty} \sup _{n \geq n_{0}}\left\{\frac{1}{\left|a_{i, n}\right|} \sum_{t=n}^{\infty} \max \left\{P_{i, t}, Q_{i, t},\left|c_{i, t}\right|\right\}\right\} \\
& =\sum_{j=m_{0}}^{\infty} \sum_{i=j}^{\infty} \sup _{n \geq n_{0}}\left\{\frac{1}{i^{5} n} \sum_{t=n}^{\infty} \max \left\{\frac{2 M^{3}\left(2 i^{3} t^{2}+2 M^{2}+1\right)}{\left(i^{3} t^{2}+N^{2}\right)^{2}}, \frac{2 M^{4}}{i^{3} t^{2}+N^{2}}, \frac{\left|i^{2}-3 t^{3}\right|}{i^{5}\left(t^{7}+1\right)}\right\}\right\} \\
& <\max \left\{4,2 M^{4}, 4 M^{3}\left(1+M^{2}\right)\right\}\left(\sum_{t=n_{0}}^{\infty} \frac{1}{t^{2}}\right) \sum_{j=m_{0}}^{\infty} \sum_{i=j}^{\infty} \frac{1}{i^{5}}<+\infty .
\end{aligned}
$$

Hence the conditions of Theorem 2.3 are fulfilled. Consequently Theorem 2.3 implies that (3.7) possesses uncountably many bounded positive solutions in $A(N, M)$. On the other hand, for any $L \in(3 N+(3 / 2) M, 2 M+(2 / 3) N)$, there exist $\theta \in(0,1)$ and $m_{1} \geq m_{0}+\tau_{0}+|\alpha|, n_{1} \geq$ $n_{0}+\sigma_{0}+|\beta|$ such that the Mann iterative sequence with errors $\{x(s)\}_{s \geq 0}$ generated by (2.25) converges to a bounded positive solution $x \in A(N, M)$ of (3.7) and has the error estimate (2.7), where $\{\gamma(s)\}_{s \geq 0}$ is an arbitrary sequence in $A(N, M),\{\alpha(s)\}_{s \geq 0}$ and $\{\beta(s)\}_{s \geq 0}$ are any sequences in $[0,1]$ satisfying $(2.8)$ and $(2.9)$.

\section{Acknowledgment}

The third author was supported by research funds from Dong-A University. 


\section{References}

[1] R. P. Agarwal, S. R. Grace, and D. O'Regan, "Nonoscillatory solutions for discrete equations," Computers \& Mathematics with Applications, vol. 45, no. 6-9, pp. 1297-1302, 2003.

[2] J. Cheng, "Existence of a nonoscillatory solution of a second-order linear neutral difference equation," Applied Mathematics Letters, vol. 20, no. 8, pp. 892-899, 2007.

[3] L. Kong, Q. Kong, and B. Zhang, "Positive solutions of boundary value problems for third-order functional difference equations," Computers \& Mathematics with Applications, vol. 44, no. 3-4, pp. 481489, 2002.

[4] B. Karpuz and Ö. Öcalan, "Further oscillation criteria for partial difference equations with variable coefficients," Computers \& Mathematics with Applications, vol. 59, no. 1, pp. 55-63, 2010.

[5] Z. Liu, S. M. Kang, and J. S. Ume, "Existence of uncountably many bounded nonoscillatory solutions and their iterative approximations for second order nonlinear neutral delay difference equations," Applied Mathematics and Computation, vol. 213, no. 2, pp. 554-576, 2009.

[6] Z. Liu, Y. Xu, and S. M. Kang, "Global solvability for a second order nonlinear neutral delay difference equation," Computers $\mathcal{E}$ Mathematics with Applications, vol. 57, no. 4, pp. 587-595, 2009.

[7] M. Migda and J. Migda, "Asymptotic properties of solutions of second-order neutral difference equations," Nonlinear Analysis, Theory, Methods and Applications, vol. 63, no. 5-7, pp. e789-e799, 2005.

[8] R. N. Rath, J. G. Dix, B. L. S. Barik, and B. Dihudi, "Necessary conditions for the solutions of second order non-linear neutral delay difference equations to be oscillatory or tend to zero," International Journal of Mathematics and Mathematical Sciences, vol. 2007, Article ID 60907, 16 pages, 2007.

[9] P. J. Y. Wong, "Eventually positive and monotonely decreasing solutions of partial difference equations," Computers $\mathcal{E}$ Mathematics with Applications, vol. 35, no. 4, pp. 35-58, 1998.

[10] P. J. Y. Wong and R. P. Agarwal, "Oscillation criteria for nonlinear partial difference equations with delays," Computers E Mathematics with Applications, vol. 32, no. 6, pp. 57-86, 1996.

[11] P. J. Y. Wong and R. P. Agarwal, "Nonexistence of unbounded nonoscillatory solutions of partial difference equations," Journal of Mathematical Analysis and Applications, vol. 214, no. 2, pp. 503-523, 1997.

[12] J. Yan and B. Liu, "Asymptotic behavior of a nonlinear delay difference equation," Applied Mathematics Letters, vol. 8, no. 6, pp. 1-5, 1995.

[13] C. Yang and P. Weng, "Green functions and positive solutions for boundary value problems of thirdorder difference equations," Computers \& Mathematics with Applications, vol. 54, no. 4, pp. 567-578, 2007.

[14] J. Yang and Y. J. Zhang, "Frequent oscillatory solutions of a nonlinear partial difference equation," Journal of Computational and Applied Mathematics, vol. 224, no. 2, pp. 492-499, 2009.

[15] L. S. Liu, "Ishikawa and Mann iterative process with errors for nonlinear strongly accretive mappings in Banach spaces," Journal of Mathematical Analysis and Applications, vol. 194, no. 1, pp. 114-125, 1995. 


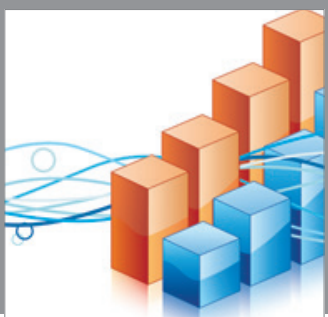

Advances in

Operations Research

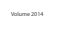

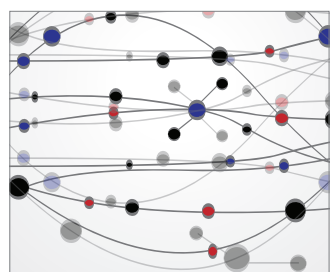

\section{The Scientific} World Journal
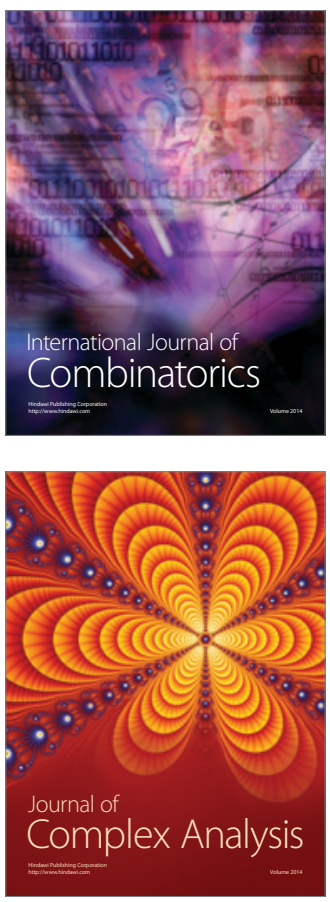

International Journal of

Mathematics and

Mathematical

Sciences
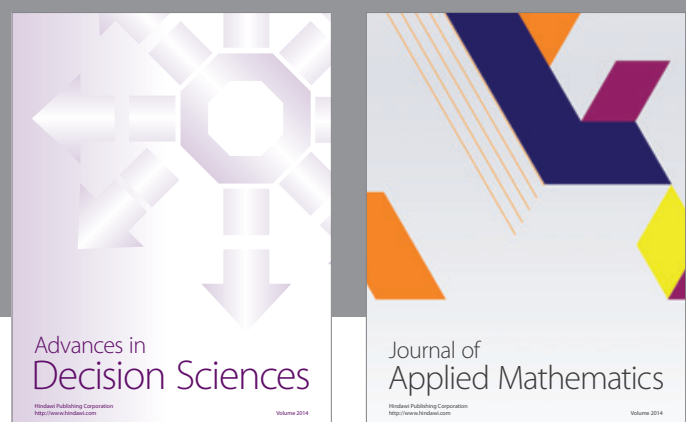

Journal of

Applied Mathematics
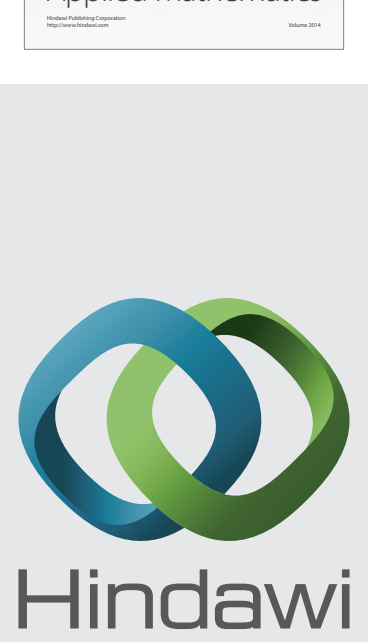

Submit your manuscripts at http://www.hindawi.com
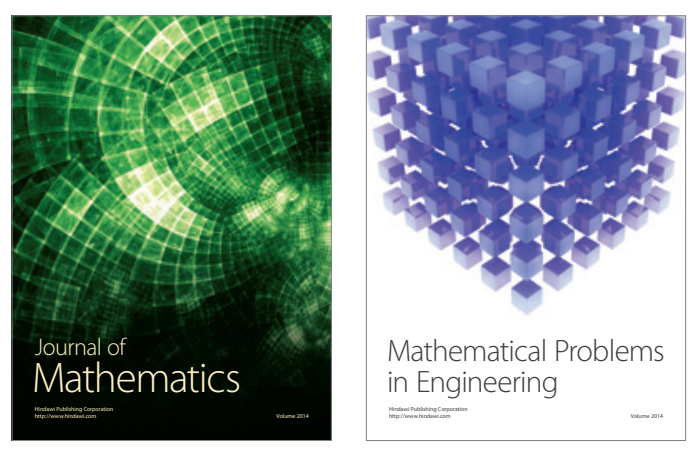

Mathematical Problems in Engineering
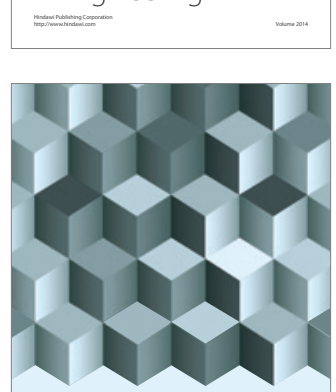

Journal of

Function Spaces
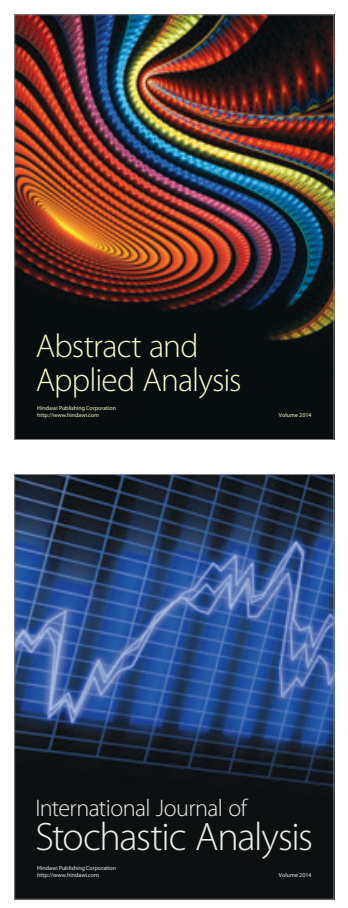

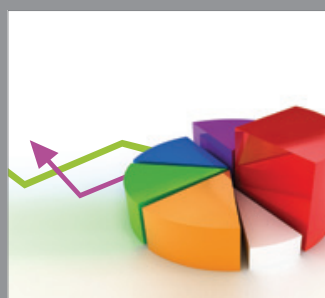

ournal of

Probability and Statistics

Promensencen
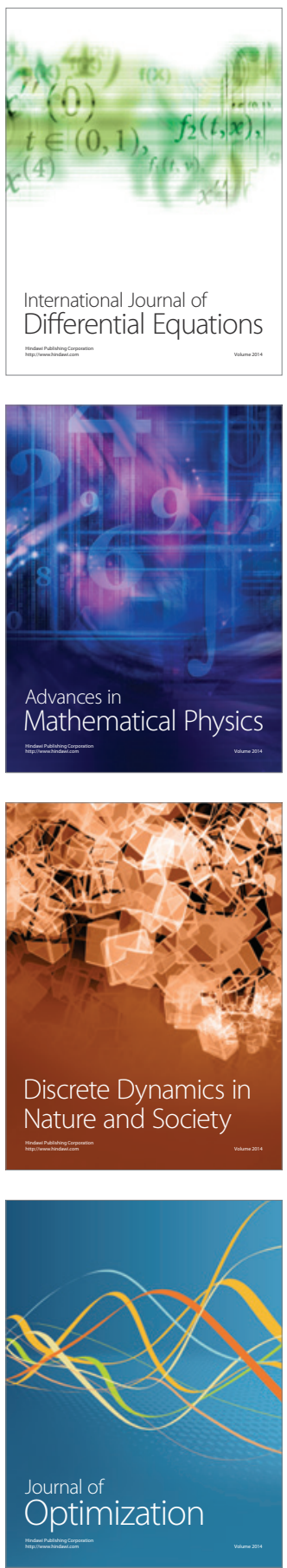